\title{
Kv1.3 modulates neuroinflammation and neurodegeneration in Parkinson's disease
}

\author{
Souvarish Sarkar, ${ }^{1}$ Hai M. Nguyen, ${ }^{2}$ Emir Malovic, ${ }^{1}$ Jie Luo, ${ }^{1}$ Monica Langley, ${ }^{1}$ Bharathi N. Palanisamy, ${ }^{1}$ Neeraj Singh, ${ }^{1}$ Sireesha Manne, ${ }^{1}$ \\ Matthew Neal, ${ }^{1}$ Michelle Gabrielle, ${ }^{3}$ Ahmed Abdalla, ${ }^{1}$ Poojya Anantharam, ${ }^{4}$ Dharmin Rokad, ${ }^{1}$ Nikhil Panicker, ${ }^{1}$ Vikrant Singh, ${ }^{2}$ \\ Muhammet Ay, ${ }^{1}$ Adhithiya Charli,, Dilshan Harischandra, ${ }^{1}$ Lee-Way Jin, ${ }^{5}$ Huajun Jin, ${ }^{1}$ Srikant Rangaraju, ${ }^{6}$ Vellareddy Anantharam, ${ }^{1}$ \\ Heike Wulff, ${ }^{2}$ and Anumantha G. Kanthasamy' \\ 1Parkinson Disorders Research Laboratory, Department of Biomedical Sciences, lowa State University (ISU), Ames, lowa, USA. ²Department of Pharmacology, School of Medicine, UCD, Davis, California, USA. \\ ${ }^{3}$ Department of Biochemistry, University of Western Ontario, London, Ontario, Canada. ${ }^{4}$ Department of Veterinary Diagnostic and Production Animal Medicine, Veterinary Medicine Building, ISU, Ames, lowa, USA. \\ 5M.I.N.D. Institute, Alzheimer's Disease Center, Department of Pathology and Laboratory Medicine, UCD, Davis, California, USA. 'Department of Neurology, Emory University School of Medicine, Atlanta, Ceorgia, USA.
}

\begin{abstract}
Characterization of the key cellular targets contributing to sustained microglial activation in neurodegenerative diseases, including Parkinson's disease (PD), and optimal modulation of these targets can provide potential treatments to halt disease progression. Here, we demonstrated that microglial Kv1.3, a voltage-gated potassium channel, was

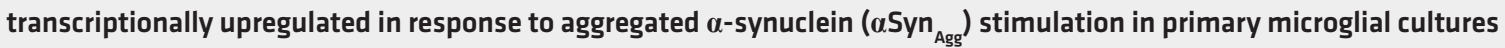
and animal models of PD, as well as in postmortem human PD brains. Patch-clamp electrophysiological studies confirmed that the observed Kv1.3 upregulation translated to increased Kv1.3 channel activity. The kinase Fyn, a risk factor for PD, modulated transcriptional upregulation and posttranslational modification of microglial Kv1.3. Multiple state-ofthe-art analyses, including Duolink proximity ligation assay imaging, revealed that Fyn directly bound to Kv1.3 and posttranslationally modified its channel activity. Furthermore, we demonstrated the functional relevance of Kv1.3 in augmenting the neuroinflammatory response by using Kv1.3-KO primary microglia and the Kv1.3-specific small-molecule inhibitor PAP-1, thus highlighting the importance of Kv1.3 in neuroinflammation. Administration of PAP-1 significantly inhibited neurodegeneration and neuroinflammation in multiple animal models of PD. Collectively, our results imply that Fyn-dependent regulation of Kv1.3 channels plays an obligatory role in accentuating the neuroinflammatory response in PD and identify Kv1.3 as a potential therapeutic target for PD.
\end{abstract}

\section{Introduction}

Parkinson's disease (PD) is a complex neurodegenerative disorder that is characterized mainly by the slow and progressive loss of dopaminergic neurons in the substantia nigra pars compacta $(\mathrm{SNpc})$ region of the brain. This progressive loss of neurons is also associated with the release of a misfolded and aggregated protein, $\alpha$-synuclein ( $\alpha \mathrm{Syn})$, which is predominantly expressed in neurons throughout the brain. Recent studies have further shown the loss of extrastriatal neurons and the presence of nonmotor deficits in PD $(1,2)$. Though the underlying cause of this neuronal loss is not completely understood, postmortem studies have implicated nigral inflammation characterized by persistent and excessive microgliosis and astrogliosis in the pathophysiology of PD. The hypothesis that neuroinflammation is critical to PD progression is

Conflict of interest: ACK and VA have an equity interest in PK Biosciences Corporation located in Ames, lowa, USA. The terms of this arrangement have been reviewed and approved by ISU in accordance with its conflict-of-interest policies. HW is an inventor on a 2004 University of California patent claiming PAP-1 as an immunosuppressant (5-Phenoxyalkoxypsoralens and methods for selective inhibition of the voltage-gated Kv1.3 potassium channel, patent no. US7557138B2).

Copyright: (c) 2020, American Society for Clinical Investigation.

Submitted: January 9, 2020; Accepted: April 29, 2020; Published: June 29, 2020.

Reference information: J Clin Invest. 2020;130(8):4195-4212.

https://doi.org/10.1172/JCl136174. further supported by evidence from cell cultures, animal models, and postmortem human tissue analysis (3-6). Currently, there are no treatments to prevent the progression of PD, and the only available therapies are directed toward mitigating its symptoms.

Gene mutations (e.g., PARK1/4) can lead to misfolding and aggregation of $\alpha$ Syn, the accumulation of which into Lewy bodies and neurites is the hallmark of both sporadic and genetically inherited PD. Interaction between aggregated $\alpha \operatorname{Syn}\left(\alpha \mathrm{Syn}_{\mathrm{Agg}}\right)$ and microglial immune receptors, such as the pattern recognition receptors TLR2 and CD36, on microglial plasma membranes can lead to microglial activation (7). Furthermore, $\alpha$ Syn has been demonstrated to induce IL- $1 \beta$ production in monocytes $(8,9)$ and microglia (10). However, much remains unknown regarding the key signaling pathways involved in either $\alpha \mathrm{Syn}_{\mathrm{Agg}}$-mediated neuroinflammation in the nigral dopaminergic system $(11,12)$ or in chronic inflammation in microglia.

A voltage-gated potassium channel with the biophysical properties of $\operatorname{Kv1.3}(13,14)$ was first described in T cells $(15,16)$ but has since also been identified in B lymphocytes and macrophages, retinal ganglion cells, microglia, and neurons, where Kv1.3 is mostly part of heteromultimers with other Kv1 family members (17). Like the other 40 voltage-gated potassium channels found in humans, Kv1.3 consists of $4 \alpha$ subunits, each with 6 transmembrane segments (S1-S6) and a voltage sensor in the S4 segment that is 
A

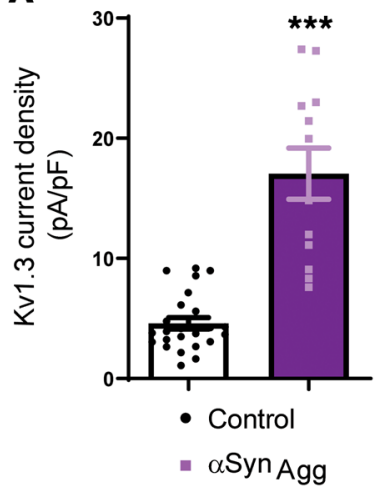

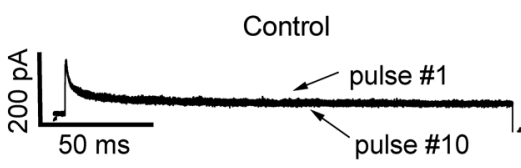

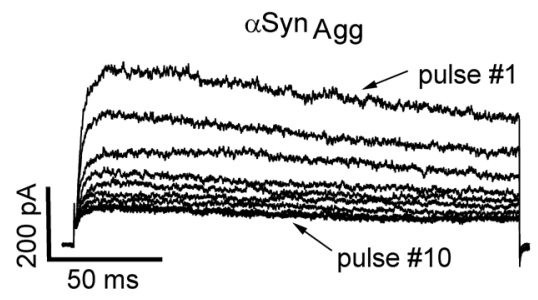

B

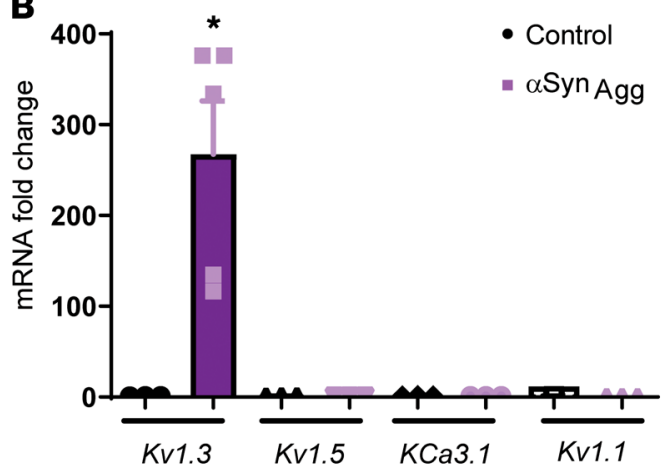

C
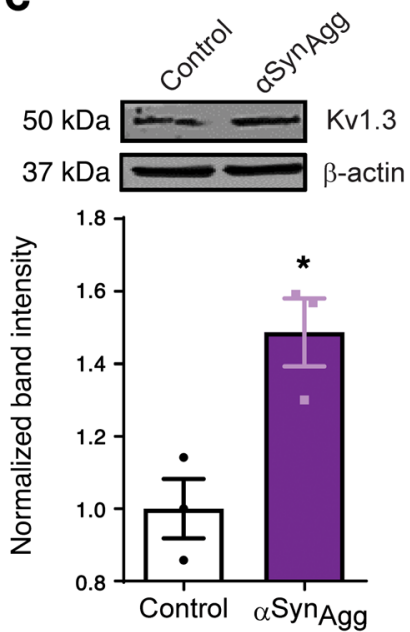

E

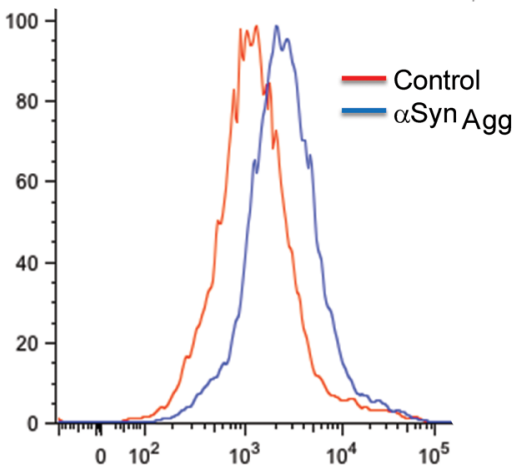

D
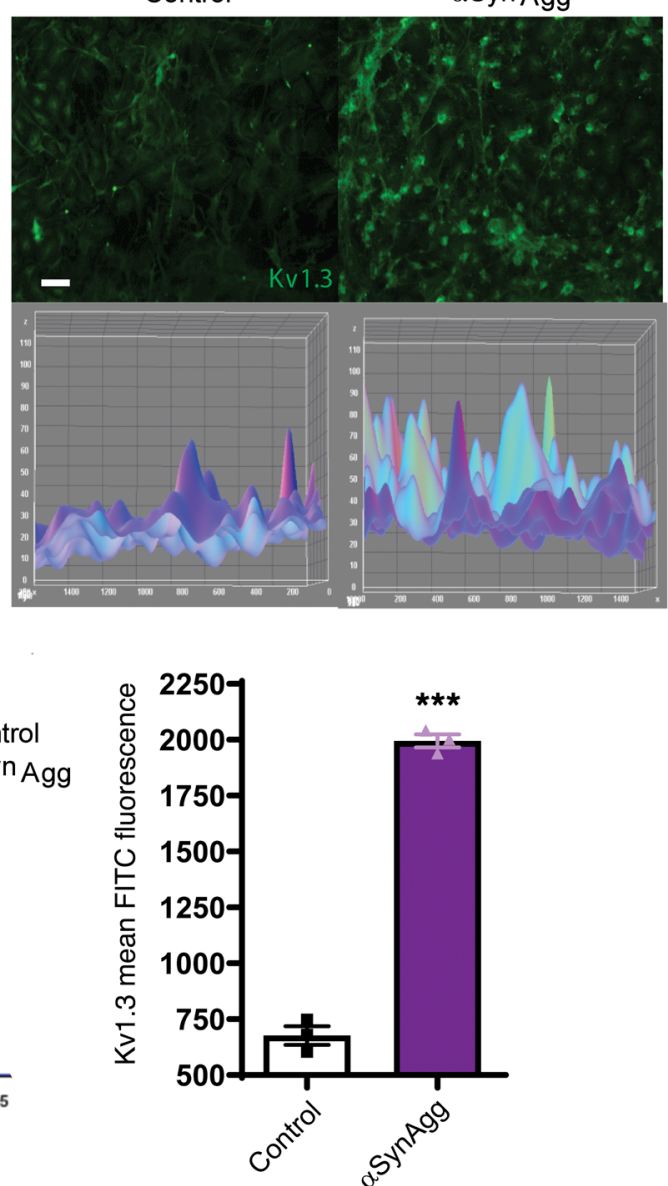

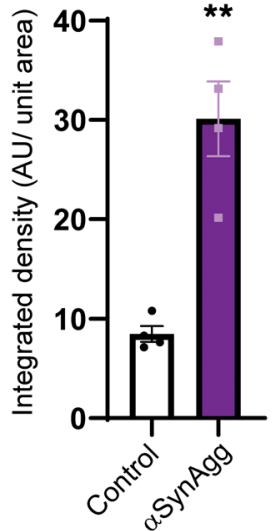

$\mathbf{F}$

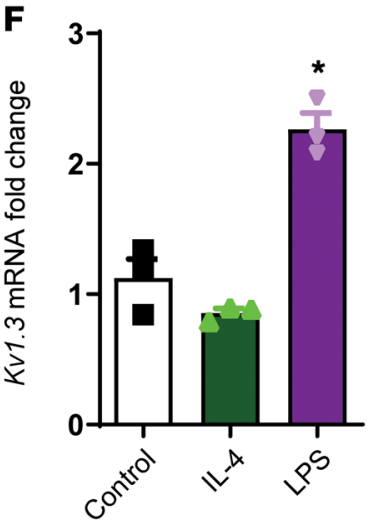

Figure 1. Upregulated expression of the potassium channel Kv1.3 upon aggregated $\alpha$ Syn stimulation in microglial cells in vitro. (A) Whole-cell patch-clamp recordings of PMCs treated with $1 \mu \mathrm{M} \alpha \mathrm{Syn}_{\mathrm{Agg}}$ for 24-48 hours, showing $\alpha \mathrm{Syn}_{\mathrm{Agg}}$-induced increased Kv1.3 activity (control $n=24$ and $\alpha \mathrm{Syn}_{\mathrm{Agg}} n=12$ ). Kv1.3 was identified by its characteristic use dependence, which was revealed when applying a train of ten $200-\mathrm{ms}$ pulses from -80 to $40 \mathrm{mV}$ at 1 -second intervals (1 Hz). (B) qRTPCR showing that $\alpha \mathrm{Syn}_{\mathrm{Agg}}$ induced Kv1.3 mRNA expression without significantly altering other potassium channels. (C) Western blot of $\alpha \mathrm{Syn}_{\mathrm{Agg}}$-induced Kv1.3 protein expression in PMCs. (D) ICC of $\alpha \mathrm{Syn}_{\mathrm{Agg}}$-induced Kv1.3 protein expression in PMCs. Scale bar: $100 \mu \mathrm{m}$. (E) Flow cytometric analysis of immortalized MMCs treated with $1 \mu \mathrm{M} \alpha \mathrm{Syn}_{\mathrm{Agg}}$ for 24 hours, showing $\alpha \mathrm{Syn}_{\mathrm{Agg}}$-induced Kv1.3 surface expression. (F) qRT-PCR of human microglia treated with LPS $(1 \mu \mathrm{g} / \mathrm{mL})$ and IL-4 (20 ng/mL) for 6 hours, showing LPS-induced KV1.3 expression. A 1-way ANOVA was used to compare multiple groups. In most cases, Tukey's post hoc analysis was applied in B and F. A 2-tailed Student's $t$ test was used for all other figures when comparing 2 groups. Each dot on the bar graphs represents a biological replicate. Data are presented as the mean \pm SEM, with 3-5 biological replicates from 2-3 independent experiments unless otherwise noted. ${ }^{*} P \leq 0.05,{ }^{* *} P<0.01$, and ${ }^{* * *} P<0.001$.

responsible for opening the channel in response to membrane depolarization. Kv1.3-KO mice are resistant to diet-induced obesity $(18,19)$ and experimental autoimmune encephalomyelitis $(20)$ and also exhibit enhanced olfaction (21). These findings reveal the diverse functions of this voltage-gated channel, though its investigation has mostly been limited to autoimmune diseases. Kv1.3 also plays an important role in microglia-mediated neuronal cell death (22) and inflammatory cytokine production by microglia in 

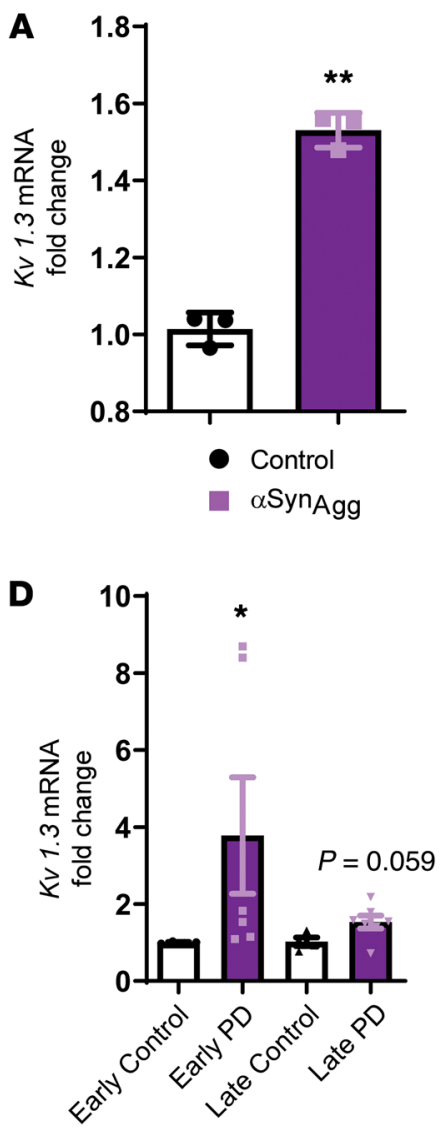
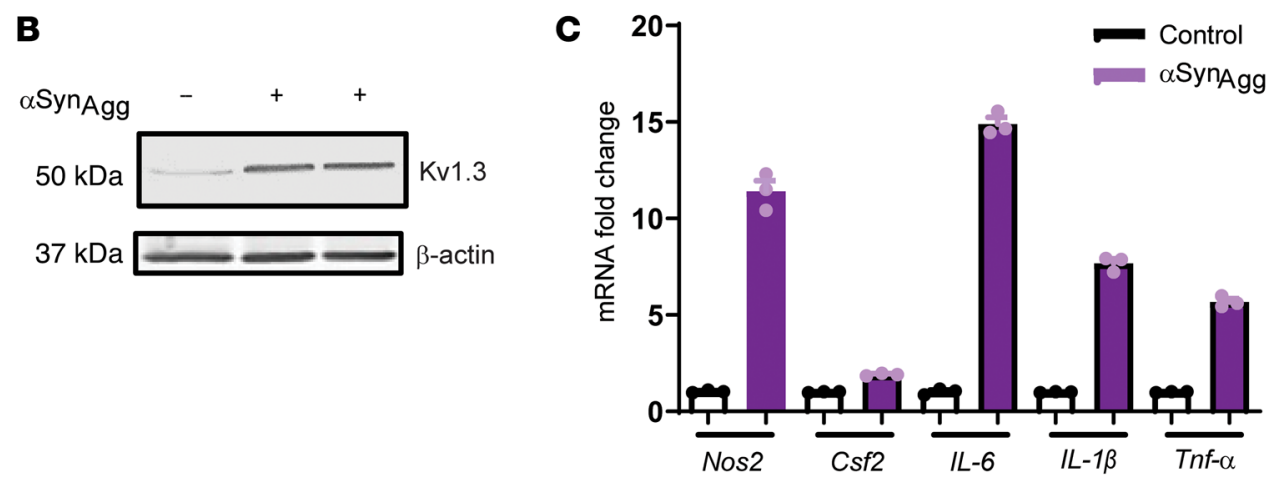

Figure 2. Upregulated expression of the potassium channel Kv1.3 upon aggregated $\alpha$ Syn stimulation in ex vivo slices and $B$ cells derived from patients with PD. (A) Midbrain slice cultures were treated with $1 \mu \mathrm{M}$ $\alpha \mathrm{Syn}_{\mathrm{Agg}}$ for 24 hours. qRT-PCR shows upregulated Kv1.3 mRNA expression. (B) Western blot shows upregulated Kv1.3 protein level in midbrain slice cultures treated with $1 \mu \mathrm{M} \alpha \mathrm{Syn}_{\mathrm{Agg}}$ for 24 hours. (C) qRT-PCR of midbrain slice cultures treated with $1 \mu \mathrm{M} \alpha \mathrm{S}_{\mathrm{yn}}$ for 24 hours, revealing upregulation of the proinflammatory factors Nos2, Csf2, IL-6, IL-1B, and Tnfa. (D) qRT-PCR shows increased Kv1.3 mRNA expression in B cell lymphocytes isolated from patients with PD compared with expression in B cell lymphocytes from agematched controls. (E) Whole-cell patch clamping of B cell lymphocytes isolated from patients with PD showed higher Kv1.3 channel activity compared with that observed in age-matched controls ( $n=3$ control and $n=3 \mathrm{PD}$ ). A 1-way ANOVA was used to compare multiple groups in $\mathbf{C}$ and $\mathbf{D}$. Tukey's post hoc analysis was applied. A 2-tailed Student's $t$ test was used to compare 2 groups. Each dot on the bar graphs represents a biological replicate. Data are presented as the mean \pm SEM, with 3-7 biological replicates from 2-3 independent experiments unless otherwise indicated. ${ }^{*} P \leq 0.05$ and ${ }^{*} P<0.01$.

vitro and in vivo $(23,24)$. Recently, Kv1.3 was shown to be upregulated in the postmortem brains of patients with Alzheimer's disease (AD) (25) and has been suggested to act as a pharmacological target for suppressing AD-associated neuroinflammation, based on work in AD mouse models (26). Interestingly, Kv1.3 contains multiple proline-rich sequences to which Src homology (SH) domains can bind, and recently, our laboratory showed that Fyn, a Src family kinase, is upregulated in PD models as well as in human PD nigral tissues and plays an important role in microglial inflammation $(10,27)$. Thus, we hypothesized that Fyn and Kv1.3 interact to augment the neuroinflammatory response, thereby regulating neurodegeneration in PD.

In this study, we investigated the role of microglial Kv1.3 in PD pathology and the transcriptional and posttranslational modifications of this channel in PD models. Our in vitro studies showed that Kv1.3 was highly upregulated in microglial cells treated with the PD-specific antigen $\alpha \mathrm{Syn}_{\mathrm{Agg}}$. Kv1.3 was also upregulated in multiple animal models of PD and in postmortem human PD brains. Kv1.3 upregulation was not confined to the brain in humans, as lymphocytes isolated from patients with PD contained higher levels of Kv1.3 than did those obtained from control subjects, making it a potential biomarker. Furthermore, we report that Fyn could regulate Kv1.3 both transcriptionally and posttranslationally, thereby modulating its functional activity. Last, PAP-1, a small-molecule inhibitor of Kv1.3 that can cross the blood-brain barrier $(26,28)$, was able to reduce neuroinflammation and neurodegeneration in cell cultures and animal models of PD. We believe our findings have significant translational implications for the therapeutic treatment of PD.

\section{Results}

Upregulation of Kv1.3 potassium channel expression upon $\alpha S_{\text {Syn }}$ Agg $_{\text {Stim- }}$ ulation in microglial cells and PD immune cells. Since Kv1.3 expression has been reported to increase in cultured microglia following stimulation with oligomeric amyloid- $\beta$ and in the brains of $\mathrm{AD}$ mouse models and patients with $\operatorname{AD}(25,26)$, we tested whether $\alpha \mathrm{Syn}_{\mathrm{Agg}}$, which contributes to chronic neuroinflammation by activating microglia in $\operatorname{PD}(10,27,29)$, induces a similar increase in Kv1.3 expression. We therefore established mixed glial cultures from neonatal mice and treated primary microglia (PMCs) with 1 $\mu \mathrm{M} \alpha \mathrm{Syn}_{\mathrm{Agg}}$ for 24 to 48 hours. Whole-cell patch clamping revealed that $\alpha \mathrm{Syn}_{\mathrm{Agg}}$ treatment increased functional Kv1.3 channel activity (Figure 1A). Parallel quantitative reverse transcription PCR (qRT$\mathrm{PCR}$ ) analysis showed that $\alpha \mathrm{Syn}_{\mathrm{Agg}}$ most significantly induced $\mathrm{Kv} 1.3$ mRNA expression compared with other potassium channels such as KCa3.1, Kv1.1, or Kv1.5 (Figure 1B). Likewise, the Kv1.3 protein level also increased, as revealed by Western blot analysis (Figure 1C) and immunocytochemistry (ICC) (Figure 1D). Furthermore, flow cytometric analysis using a FITC-labeled Kv1.3 antibody against an extracellular epitope showed that $\alpha \mathrm{Syn}_{\mathrm{Agg}}$ increased the surface expression of Kv1.3 (Figure 1E) in PMCs, in keeping with the observed increases in current density observed by electrophysiology (Figure 1A). Next, we corroborated each of our PMC findings using a line of mouse microglial cells (MMCs) for qRT-PCR (Supplemental Figure 1A; supplemental material available online with this article; https://doi.org/10.1172/JCI136174DS1), ICC (Supplemental Figure 1B), and Western blot (Supplemental Figure 1C) analyses. Moreover, whole-cell patch clamping confirmed that 
treatment with the potent microglia activator LPS also upregulated Kv1.3 channel activity in PMCs (Supplemental Figure 1D), and flow cytometry revealed increased surface expression of Kv1.3 in MMCs (Supplemental Figure 1E), indicating that the observed upregulation of Kv1.3 may play a role in mediating microglial activation. After treating the MMCs with LPS $(1 \mu \mathrm{g} / \mathrm{mL})$ and IL-4 $(20 \mathrm{ng} / \mathrm{mL})$ for 6 hours to induce classical and alternative microglial activation, respectively, we observed that LPS induced Kv1.3 mRNA expression, whereas IL-4 did not alter Kv1.3 expression (Figure 1F), further validating the finding that this voltage-gated potassium channel is related to the classical, proinflammatory activation of microglia (23). Following our in vitro studies of microglial cultures, we next adopted an ex vivo organotypic midbrain slice culture model, wherein a 24-hour exposure to $1 \mu \mathrm{M} \alpha \mathrm{Syn}_{\mathrm{Agg}}$ significantly induced the expression of Kv1.3 mRNA (Figure 2A) and protein (Figure 2B) as well as mRNA expression of the proinflammatory genes Nos2, Csf2, IL-6, IL-1 $\beta$ and Tnf- $\alpha$ (Figure 2C). Next, we assessed mRNA expression of $\mathrm{Kv} 1.3$ in PD patient- and control-derived B lymphocytic cells. Interestingly, we detected a significant increase in $\mathrm{Kv1.3}$ mRNA expression in PD patient-derived B lymphocytic cells compared with expression in age-matched control cells (Figure 2D). Importantly, the increase observed in the lymphocytic cells generated from patients with early-phase PD was much more robust than that detected in late-phase PD patient-derived cells, suggesting that lymphocytic Kv1.3 could be a potential biomarker for early PD diagnosis. Last, whole-cell patch clamping revealed that lymphocytes from patients with PD had higher Kv1.3 channel activity than did B lymphocytes from age-matched controls (Figure 2E). Collectively, these findings demonstrate that inflammatory stimuli, such as $\alpha \mathrm{Syn}_{\mathrm{Agg}}$ or LPS, induce functionally relevant increases in microglial Kv1.3 protein and mRNA levels, as indicated by the enhanced channel activity in microglial cells. Given the critical role of neuroinflammation in $\mathrm{PD}$, these data suggest a possible role of Kv1.3 in PD pathogenesis and progression.

Kv1.3 channel expression is highly induced in microglial cells in experimental in vivo models of PD and in postmortem human PD brains. PD is a multifactorial disease, and to date no in vivo models can reproduce all the symptoms of PD. Hence, to better understand the potential role of Kv1.3, we used multiple animal models of PD to recapitulate various clinical and pathological aspects of the disease. One of the major pathological hallmarks of PD is protein aggregation, which can be recapitulated in the aSyn-adeno-associated virus ( $\alpha$ Syn-AAV) mouse model (30), in which $\alpha$ Syn-AAV was stereotaxically injected into the striatum, and the mice were sacrificed 6 months later (Supplemental Figure 2A). Western blot analysis revealed a substantial induction of Kv1.3 in this animal model of PD (Figure 3A).

To verify whether Kv1.3 channel expression is induced in a more gradually progressive PD model, we used the MitoPark transgenic mouse model. MitoPark mice were developed by inactivating the mitochondrial transcription factor A (TFAM) in dopaminergic neurons (31) to model the mitochondrial dysfunction exhibited by the progressively neurodegenerative Parkinsonian syndrome, with its diverse spectrum of motor and nonmotor symptoms. We recently reported the activation of the NLRP3 inflammasome in this model, demonstrating the involvement of neuroinflammatory processes in nigral dopaminergic neuronal degeneration (32). Here, we show that $K v 1.3$ gene expression was highly induced in the substantia nigra (SN) of 16- and 24-week old MitoPark mice compared with expression in age-matched littermate controls (Figure 3B). Our Western blot analysis further revealed that Kv1.3 was upregulated in the SN region in 24-weekold MitoPark mice (Figure 3C), and we confirmed that this upregulation was localized to IBA1-positive SN microglia (Figure 3D).

Next, we adopted the well-characterized subacute 1-methyl4-phenyl-1,2,3,6-tetrahydropyridine (MPTP) model of Parkinsonism, in which mice received $20 \mathrm{mg} / \mathrm{kg}$ MPTP daily for 4 consecutive days and were sacrificed on the fifth day (Supplemental Figure 2B). In line with the $\alpha$ Syn-AAV and MitoPark models, we found that MPTP substantially increased mRNA and protein expression of nigral Kv1.3 (Figure 3, E and F). Although MPTP treatment did not induce substantial $\alpha$ Syn aggregation in mouse models, a few reports have indicated that MPTP can induce $\alpha$ Syn upregulation and aggregation in nonhuman and rodent models $(33,34)$. It is likely that MPTP-induced mitochondrial dysfunction contributed to the upregulation of Kv1.3.

To further establish the clinical relevance of Kv1.3 in the Parkinsonian syndrome, we assessed Kv1.3 channel expression in postmortem human brains from patients with PD and patients with dementia with Lewy bodies (DLBs). When comparing striatal brain samples, we observed Kv1.3 mRNA levels that were higher in postmortem human PD brains than in control brains (Figure 3G). Western blot analysis of tissues from PD and age-matched controls further showed increased Kv1.3 levels in the SN (Figure 3H). Immunostaining for Kv1.3 and IBA1 further showed upregulated Kv1.3 immunoreactivity in the prefrontal cortex (Figure 3I, top panels) and SN (Figure 3I, bottom panels) of PD brain sections that was localized to IBA1-positive microglia (Supplemental Figure 2C). We also examined Kv1.3 expression in another Parkinsonism-related synucleinopathy, DLB. Histochemical analysis of postmortem DLB brains (SN region) revealed that Kv1.3 expression had substantially increased in HLA-DR-positive microglial cells (Figure 3J) compared with expression in control brains. Together, these findings demonstrate that microglial Kv1.3 is highly induced in both animal models and patients with PD.

Fyn modulates the transcriptional regulation of Kv1.3 in microglial cells through the Fyn/PKC $\delta$ kinase signaling cascade. After confirming the expression of Kv1.3 in cell culture and various animal models of PD as well as in patients with $\mathrm{PD}$, we investigated the molecular mechanism behind the transcriptional upregulation of the Kv1.3 gene. Though a recent study demonstrated epigenetic regulation of Kv1.3 (35), its transcriptional regulation is not well understood. Our in silico analysis of the Kv1.3 promoter revealed multiple NF- $\mathrm{KB}$ and SP1 binding sites (Figure 4A), and previous studies showed that SP1 phosphorylation is dependent on the p38 MAPK pathway and that p38 MAPK is usually upstream of NF- $\mathrm{BB}$

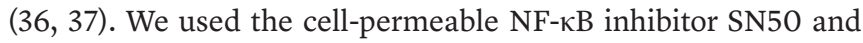
the p38 MAPK inhibitor SB203580. Both inhibitors independently attenuated $\alpha \mathrm{Syn}_{\mathrm{Agg}}$-induced $K v 1.3$ upregulation in the microglial cell line (Figure $4 \mathrm{~B}$ ). These findings suggest that NF- $\kappa \mathrm{B}$ and $\mathrm{p} 38$ MAPK signaling pathways contributed to the $\alpha \mathrm{Syn}_{\mathrm{Agg}}$-induced Kv1.3 upregulation. We previously demonstrated that Fyn acts as an upstream regulator of the NF- $\mathrm{BB}$ transcription factor by regulating the nuclear translocation of p65 (27). Fyn has also been shown 
A

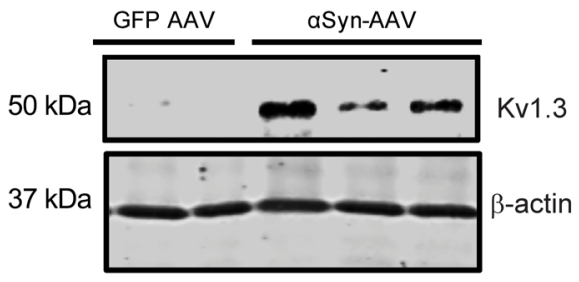

D

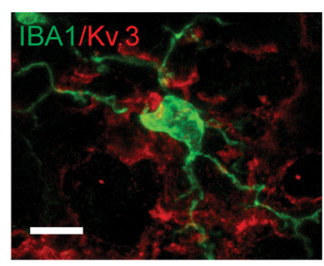

MitoPark

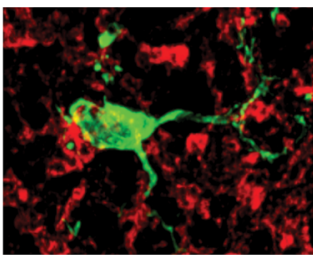

B

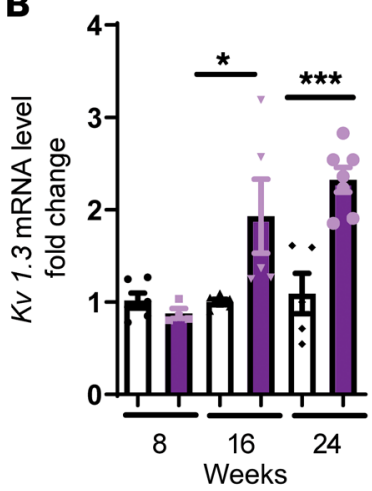

C

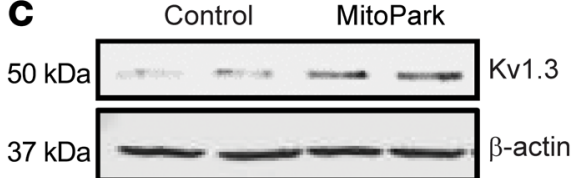

E

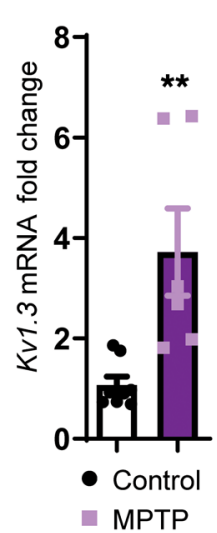$$
\text { MPTP }
$$

$\mathbf{F}$ $50 \mathrm{kDa}$

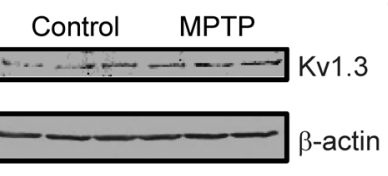

I

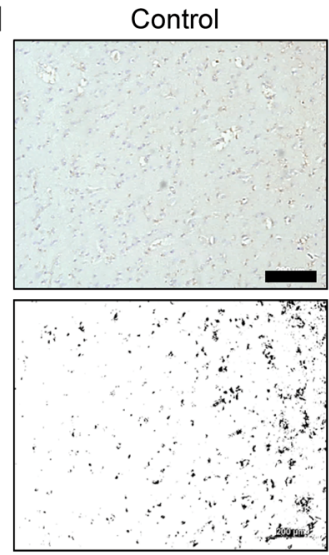

$37 \mathrm{kDa}$

$$
\text { I }
$$

Control

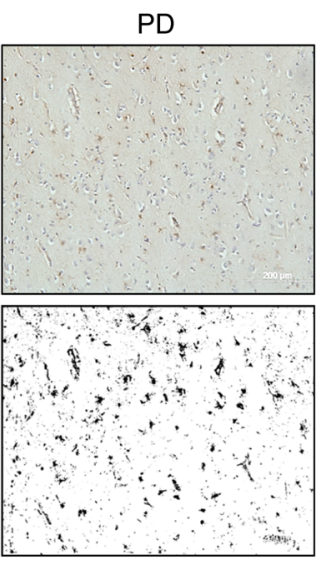

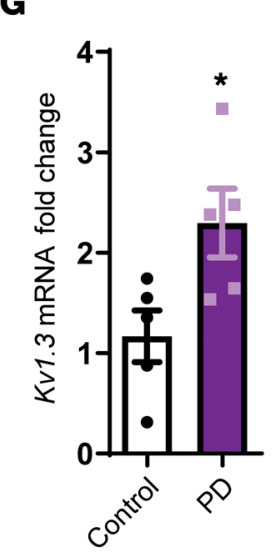

H
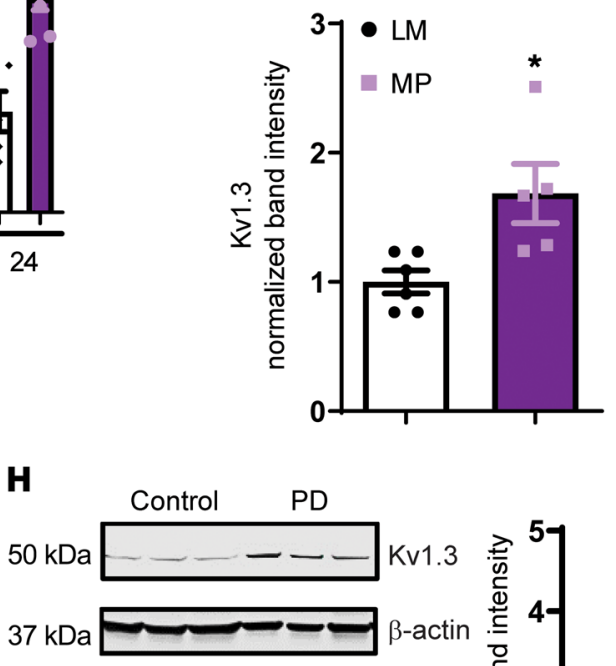

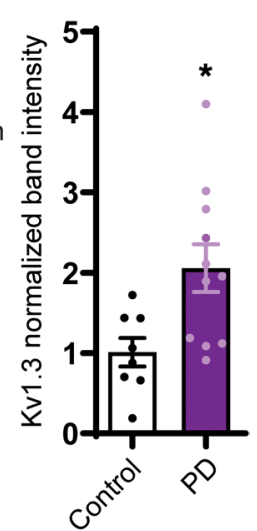

J

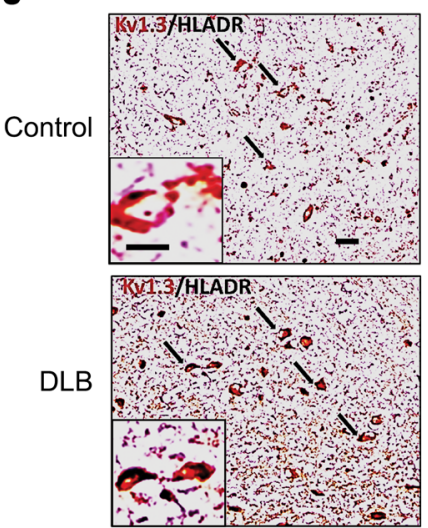

Figure 3. Kv1.3 expression is highly induced in microglial cells in experimental models of PD and postmortem PD brains. (A) Western blot showing increased Kv1.3 protein levels in the substantia nigra of the Syn-AAV mouse model of PD. (B) qRT-PCR analysis of 8- to 24-week-old nigral tissues from the MitoPark mouse model of PD showing Kv1.3 induction compared with age-matched littermate controls. (C) Western blot of 24-week-old nigral tissues from the MitoPark mouse model of PD (MP) showing induction of Kv1.3 protein expression compared with age-matched littermate control mice (LM). (D) IHC in 24-week-old nigral tissues from the MitoPark mouse model of PD showing higher Kv1.3 protein levels (red) in IBA1-positive microglial cells (green) compared with age-matched controls as revealed by their colocalization (yellow). Scale bar: $20 \mu \mathrm{m}$. (E) qRT-PCR analysis of nigral tissues from the MPTP mouse model revealing induction of Kv1.3 mRNA expression. (F) Western blot showing increased Kv1.3 protein levels in substantia nigra of the MPTP mouse model of PD. (G) qRT-PCR analysis of postmortem human PD brains showing elevated Kv1.3 mRNA expression. (H) Western blot of the SN region of postmortem human PD brain showing induction of Kv1.3 protein expression compared with age-matched controls. $n=6-8$. (I) Immunostaining revealing higher Kv1.3 levels in the prefrontal cortex of postmortem human PD brains compared with age-matched controls. Lower panel shows the deconvoluted binary image used for analysis. Three regions per brain were analyzed. Scale bar: $200 \mu \mathrm{m}$. (J) Dual DAB staining showing induction of Kv1.3 expression in HLA-DR-positive microglial cells in patients with DLBs compared with age-matched controls. Scale bars: $100 \mu \mathrm{m} ; 20 \mu \mathrm{m}$ (enlarged insets). A 1-way ANOVA was used to compare multiple groups. Tukey's post hoc analysis was applied B. A 2-tailed Student's $t$ test was used to compare 2 groups. Each dot on the bar graphs represents a biological replicate. Data are presented as the mean \pm SEM, with 3-9 biological replicates from 2-3 independent experiments unless otherwise indicated. ${ }^{*} P \leq 0.05$, ${ }^{* *} P<0.01$, and ${ }^{* * *} P<0.001$. 
A

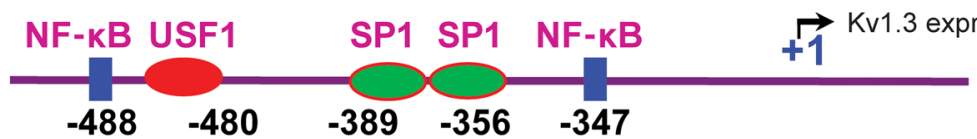

B

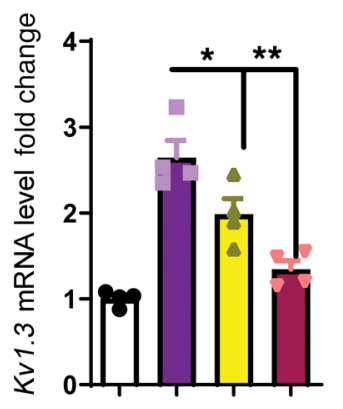

C

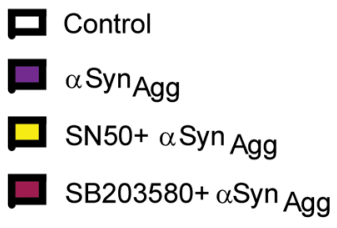

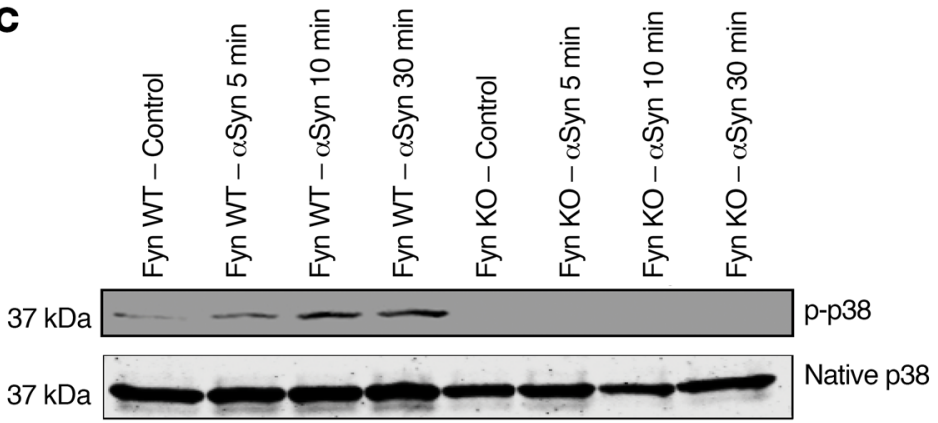

D

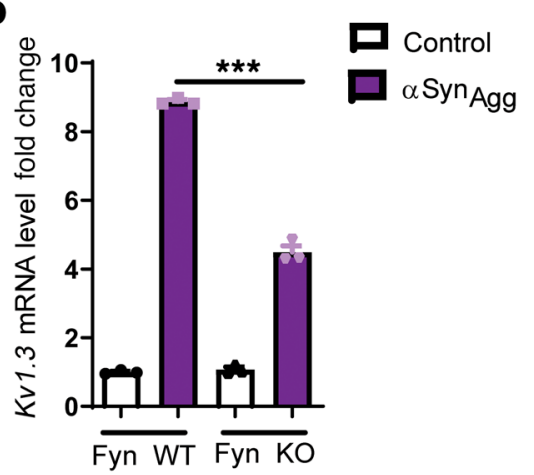

\section{E}

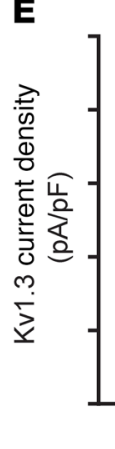

- WT Control

- WT aSynAgg

$\triangle$ Fyn KO aSynAgg

- WT LPS

- Fyn KO LPS

$\mathbf{F}$

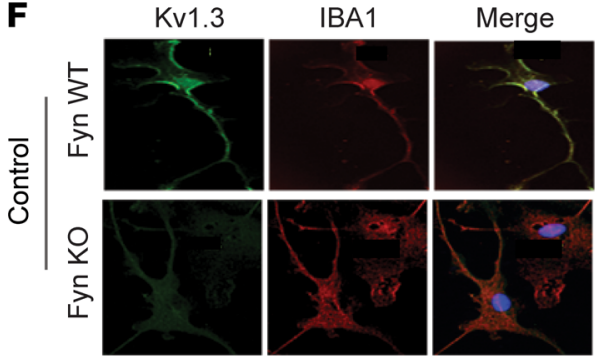

G
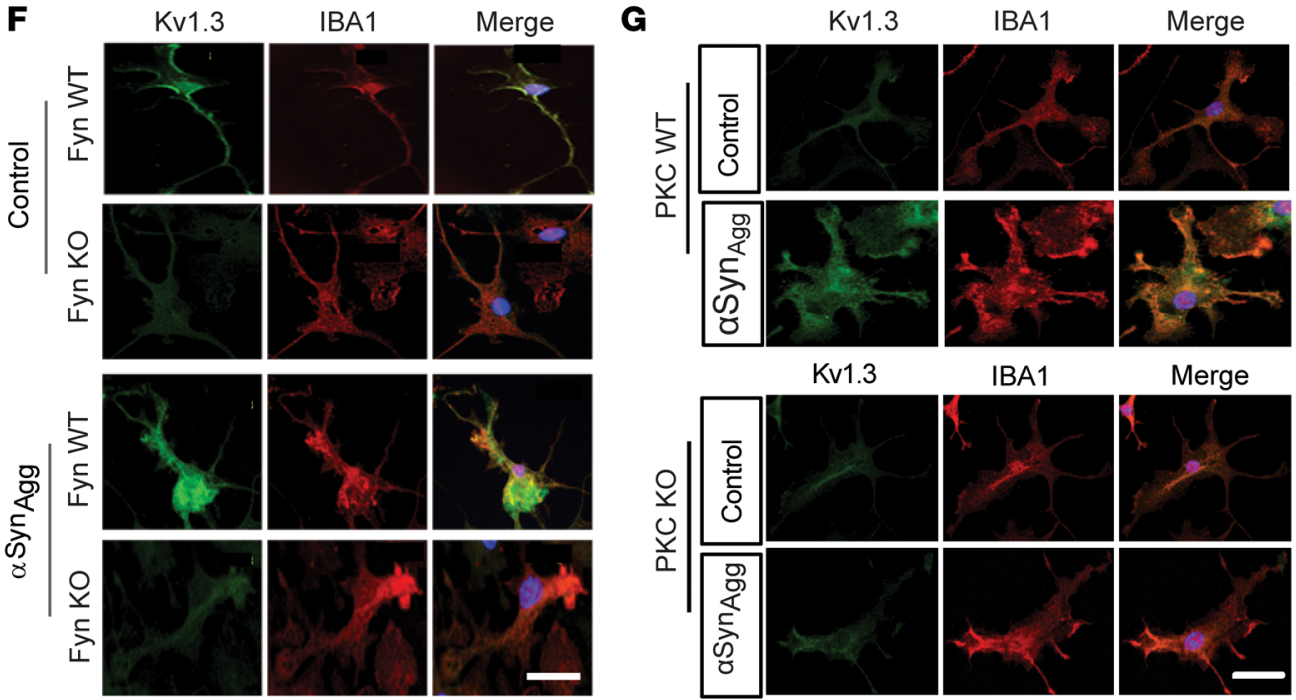

H

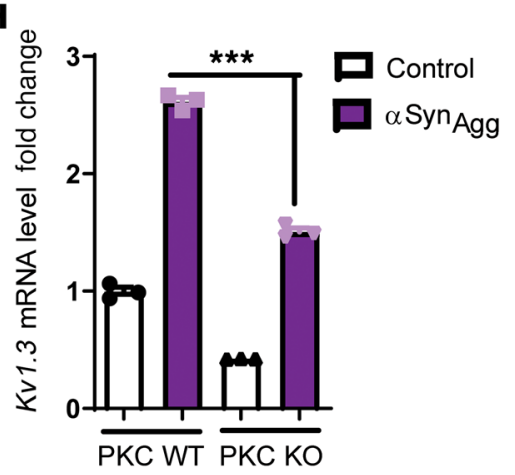

I - WT Control - WT LPS

- WT aSynAgg • PKC KO LPS

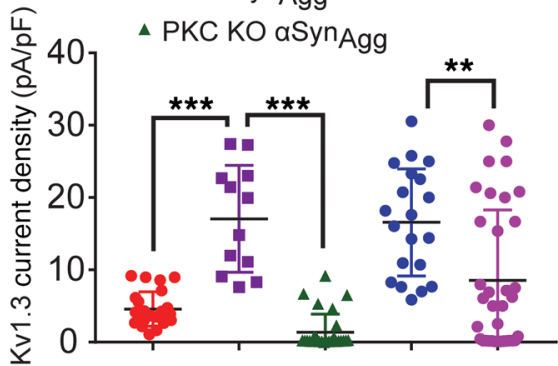


Figure 4. Fyn modulates the transcriptional regulation of Kv1.3 in microglial cells through the Fyn/PKC $\delta$ kinase signaling cascade. (A) In silico analysis of the promoter sequence of $\mathrm{Kv1} .3$ revealed probable Nf- $\mathrm{\kappa B}$ - and SP1-binding sites. (B) qRT-PCR analysis of immortalized MMCs cotreated with $\alpha \mathrm{Syn}_{\mathrm{Agg}}$ and either SN50 $(100 \mu \mathrm{g} / \mathrm{mL})$ or SB203580 $(1 \mu \mathrm{M})$, showing that both compounds attenuated $\alpha \mathrm{Syn}_{\mathrm{Agg}}$-induced Kv1.3 expression. (C) Western blot of Fyn WT and KO PMCs treated with $\alpha \mathrm{Syn}_{\mathrm{Agg}}$, showing that Fyn KO reduced the induction of the p38 MAPK pathway. (D) qRT-PCR analysis revealed that Fyn $\mathrm{KO}$ reduced $\alpha \mathrm{Syn}_{\mathrm{Agg}}$-induced Kv1.3 mRNA levels. (E) Wholecell patch-clamp recording showing that Fyn $\mathrm{KO}$ attenuated $\alpha \mathrm{Syn}_{\mathrm{Agg}}-$ and LPS-induced Kv1.3 activity compared with Fyn WT PMCs (WT control $n=24$, WT $\alpha \operatorname{Syn}_{\text {Agg }} n=12$, WT LPS $n=29$, Fyn KO $\alpha \operatorname{Syn}_{\text {Agg }} n=20$, Fyn KO LPS $n=15$ ). (F) ICC showing that Fyn KO reduced $\alpha S_{y n}{ }_{A g g}$-induced Kv1.3 protein levels in PMCs. Scale bar: $15 \mu \mathrm{m}$. (G) ICC of PMCs revealed that $\alpha$ Syn $_{\mathrm{Agg}}$-induced Kv1.3 protein expression was reduced by PKC $\delta$ KO. Scale bar: $15 \mu \mathrm{m}$. (H) qRT-PCR analysis of PMCs showing that PKC KO reduced the expression of $\alpha \mathrm{Syn}_{\mathrm{Agg}}-$ induced Kv1.3 mRNA. (I) Whole-cell patch clam recording of PMCs showing that PKC KO attenuated $\alpha \mathrm{Syn}_{\mathrm{Agg}}$ - and LPS-induced KV1.3 activity compared with PKC WT PMCs (WT control $n=24$, WT $\alpha$ Syn $_{\text {Agg }} n=12$, WT LPS $n=20$, PKC-KO $\alpha S_{y y} n_{\text {Agg }} n=29$, PKC-KO LPS $n=35$ ). Data are presented as the mean \pm SD. A 1-way ANOVA was used to compare multiple groups. In most cases, Tukey's post hoc analysis was applied. Each dot on the bar graphs represents a biological replicate. Data are presented as the mean \pm SEM, with 3-4 biological replicates from 2-3 independent experiments unless otherwise indicated. ${ }^{*} P \leq 0.05,{ }^{* *} P<0.01$, and ${ }^{* *} P<0.001$.

to modulate LPS-induced activation of the p38 MAPK pathway. Indeed, our Western blot analysis of PMC cultures revealed that knocking out Fyn in PMCs attenuated $\alpha \mathrm{Syn}_{\mathrm{Agg}}$-induced p38 phosphorylation (Figure 4C), further demonstrating that Fyn acts upstream of p38 MAPK activation following $\alpha \mathrm{Syn}_{\mathrm{Agg}}$ stimulation. Accordingly, we hypothesized that Fyn may regulate inflammagen-induced Kv1.3 upregulation. Fyn deficiency in PMCs attenuated $\alpha \mathrm{Syn}_{\mathrm{Agg}}$-induced $\mathrm{Kv1.3}$ expression, as revealed by qRT-PCR (Figure 4D) and ICC (Figure 4F) analyses. Importantly, a wholecell patch-clamp study of LPS- and $\alpha \mathrm{Syn}_{\mathrm{Agg}}$-treated microglial cells revealed that Fyn-KO microglia had lower Kv1.3 current density than did WT microglia (Figure 4E). Next, we pharmacologically inhibited Fyn in $\alpha \mathrm{Syn}_{\mathrm{Agg}}$-stimulated PMCs by cotreating them with the potent Src family kinase inhibitor saracatinib $(1 \mu \mathrm{M})$. Electrophysiological analysis revealed that saracatinib treatment reduced both LPS- and $\alpha \mathrm{Syn}_{\mathrm{Agg}}$-induced Kv1.3 channel activity, further suggesting the probable role of Fyn in modulating Kv1.3 activity (Supplemental Figure 3A). As revealed by qRT-PCR analyses, cotreatment with saracatinib reduced $\alpha \mathrm{Syn}_{\mathrm{Agg}}$-induced expression of Kv1.3 (Supplemental Figure 3B) as well as expression of the proinflammatory gene Nos2 (Supplemental Figure 3C) and the cytokines TNF- $\alpha$ and IL-1 $\beta$ (Supplemental Figure 3, E and F), without altering mRNA expression of Fyn itself (Supplemental Figure 3D).

Our group recently showed that $\mathrm{PKC} \delta$, a serine/threonine kinase involved in microglial inflammation (38), is modulated by Fyn (27). We have previously shown that Fyn can directly modulate PKC $\delta$, which can induce the activation of p38 MAPK and the nuclear translocation of p65 (27). To further show whether Kv1.3 expression is dependent on the Fyn/PKC $\delta$ signaling axis, we treated PMCs with $1 \mu \mathrm{M} \alpha \mathrm{Syn}_{\mathrm{Agg}}$ for 1 hour, followed by ICC (Figure $4 \mathrm{G}$ ) and qRT-PCR (Figure 4H), which revealed that PKC $\delta$ deficiency attenuated $\alpha \mathrm{Syn}_{\mathrm{Agg}}$-induced Kv1.3 mRNA and protein expression. Moreover, whole-cell patch clamping revealed that PKC $\delta$ deficiency reduced Kv1.3 current density in microglia treated with either $\alpha \operatorname{Syn}_{\mathrm{Agg}}(1 \mu \mathrm{M})$ for 24 hours or LPS (300 ng/mL) for 48 hours (Figure 4I). These findings collectively suggest that the Fyn/PKC $\delta$ kinase signaling cascade plays a crucial role in Kv1.3 upregulation in response to inflammatory stimuli.

Fyn modulates posttranslational modification of Kv1.3. Posttranslational modification of Kv1.3 activity and other cellular functions has been attributed to phosphorylation of serine/ threonine residues or tyrosine residues by various kinases and dephosphorylation by phosphatases (39-42). Kv1.3 has proline-rich regions in its $\mathrm{N}$-terminal and $\mathrm{C}$-terminal ends, which can bind to the Src homology domain 3 (SH3) of Src kinases. We have linked Fyn to the regulation of microglial inflammation (27), which prompted us to hypothesize that Fyn plays an important role in regulating the phosphorylation profile of Kv1.3 (43). Our multiple sequence alignment using T-Coffee software (44) to align $\mathrm{N}$-terminal Kv1.3 sequences from 7 different organisms demonstrated that Kv1.3 is highly conserved and that the tyrosine residue at 135 (187 in humans) is conserved in all species (Supplemental Figure 4). Interestingly, Western blot analyses of postmortem human brains revealed that patients with $\mathrm{PD}$ expressed significantly more pY135 Kv1.3 than age-matched controls (Figure 5A).

To verify whether Fyn directly interacts with Kv1.3, we performed co-IP assays. After treating PMCs with $\alpha \mathrm{Syn}_{\mathrm{Agg}}$ for 24 hours, Fyn and Kv1.3 were pulled down and probed for Kv1.3 and Fyn, respectively. Our immunoblot analysis revealed that Fyn interacted with Kv1.3 in $\alpha \mathrm{Syn}_{\mathrm{Agg}}$-exposed PMCs (Figure 5B). We performed a proximity ligation assay (PLA) using Duolink, which further verified the interaction of Fyn with Kv1.3 (Figure 5C). We also treated PMCs from $\mathrm{Fyn}^{+/+}$and $\mathrm{Fyn}^{-/-}$mice with $\alpha \mathrm{Syn}_{\mathrm{Agg}}$, and both Western blot (Figure 5D) and ICC (Supplemental Figure $5 \mathrm{~A}$ ) analyses revealed that the $\alpha \mathrm{Syn}_{\mathrm{Agg}}$-induced upregulation of phosphorylated Kv1.3 (p-Kv1.3) at residue 135 was diminished in $\mathrm{Fyn}^{-/-}$microglia. This finding is in line with the whole-cell patchclamp data in Figure $4 \mathrm{E}$ showing that $\alpha \mathrm{Syn}_{\mathrm{Agg}}{ }^{-}$and LPS-treated $\mathrm{Fyn}^{-1-}$ PMCs had reduced current compared with $\mathrm{Fyn}^{+/+}$cells. To add in vivo relevance, we performed IHC analysis on the previously described $\alpha$ Syn preformed fibrils $\left(\alpha \mathrm{Syn}_{\mathrm{PFF}}\right)$ model. Interestingly, injections of $\alpha \mathrm{Syn}_{\mathrm{Agg}}$ induced phosphorylation of Kv1.3 at Tyr35 in $\mathrm{Fyn}^{+/+}$mice, but this induction of phosphorylation was not evident in $\mathrm{Fyn}^{-/}$mice, further demonstrating the role of Fyn in modulating the phosphorylation of Kv1.3 at Tyr135 (Figure 5E). Furthermore, we orally administered the Fyn inhibitor saracatinib to MitoPark mice aged 8-16 weeks every other day and then examined the phosphorylation status of Kv1.3. IHC analysis revealed that Fyn inhibition decreased p-Kv1.3 (Tyr135) in the SN region of 16-weekold MitoPark mice (Figure 5F). These data collectively suggest that Fyn directly phosphorylates Kv1.3 at residue 135 in PD models.

To further evaluate the role of this phosphorylation at residue 135 in $\alpha$ Syn $_{\mathrm{Agg}}$-stimulated inflammatory responses, MMCs were transiently transfected with a Kv1.3 WT plasmid or a mutant plasmid, whereby the tyrosine residue at 135 was mutated to alanine. Following transfection, the cells were treated with $1 \mu \mathrm{M} \alpha \mathrm{Syn}_{\mathrm{Agg}}$ for 24 hours. Western blot analysis revealed that ectopic expression of the Kv1.3 mutant attenuated $\alpha \mathrm{Syn}_{\mathrm{Agg}}-$ induced upregulation of Kv1.3 phosphorylation (Supplemental Figure 5B), whereas the qRT-PCR, Griess, and Luminex anal- 
A
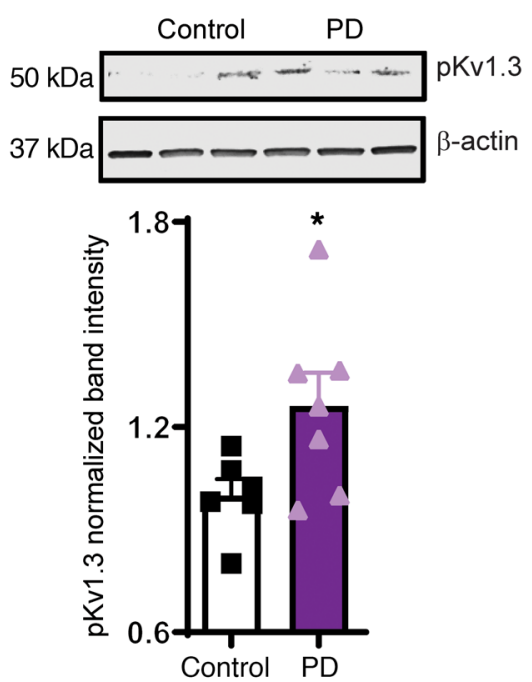

E
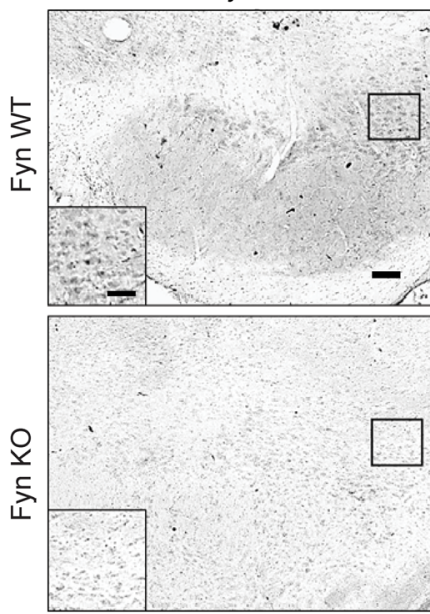

G

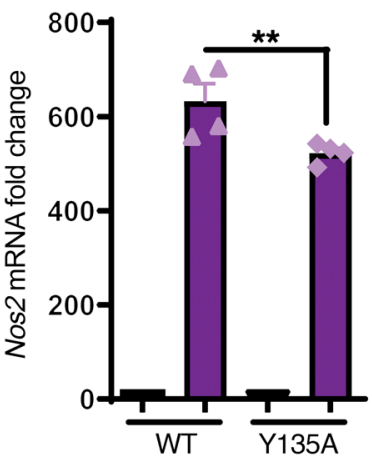

H
B

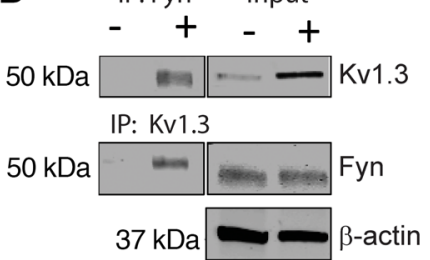

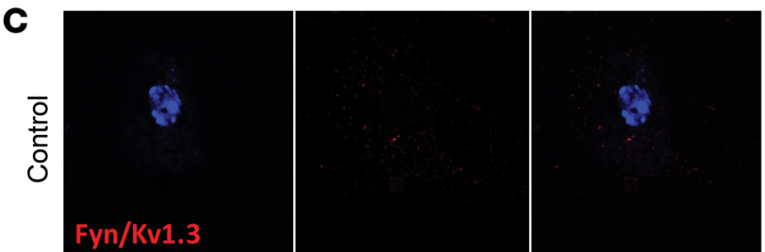

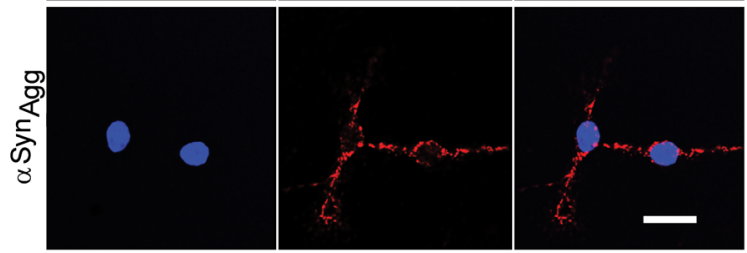

D

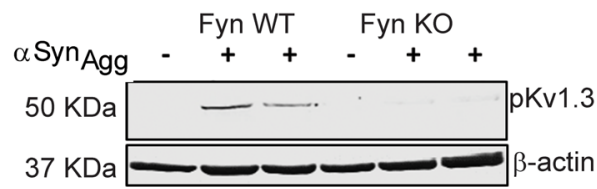

Injected

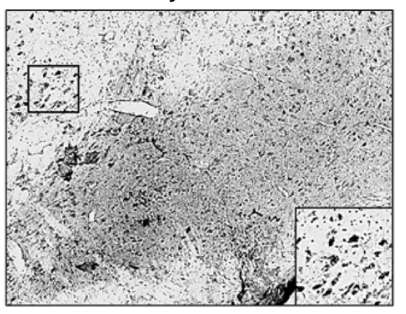

F
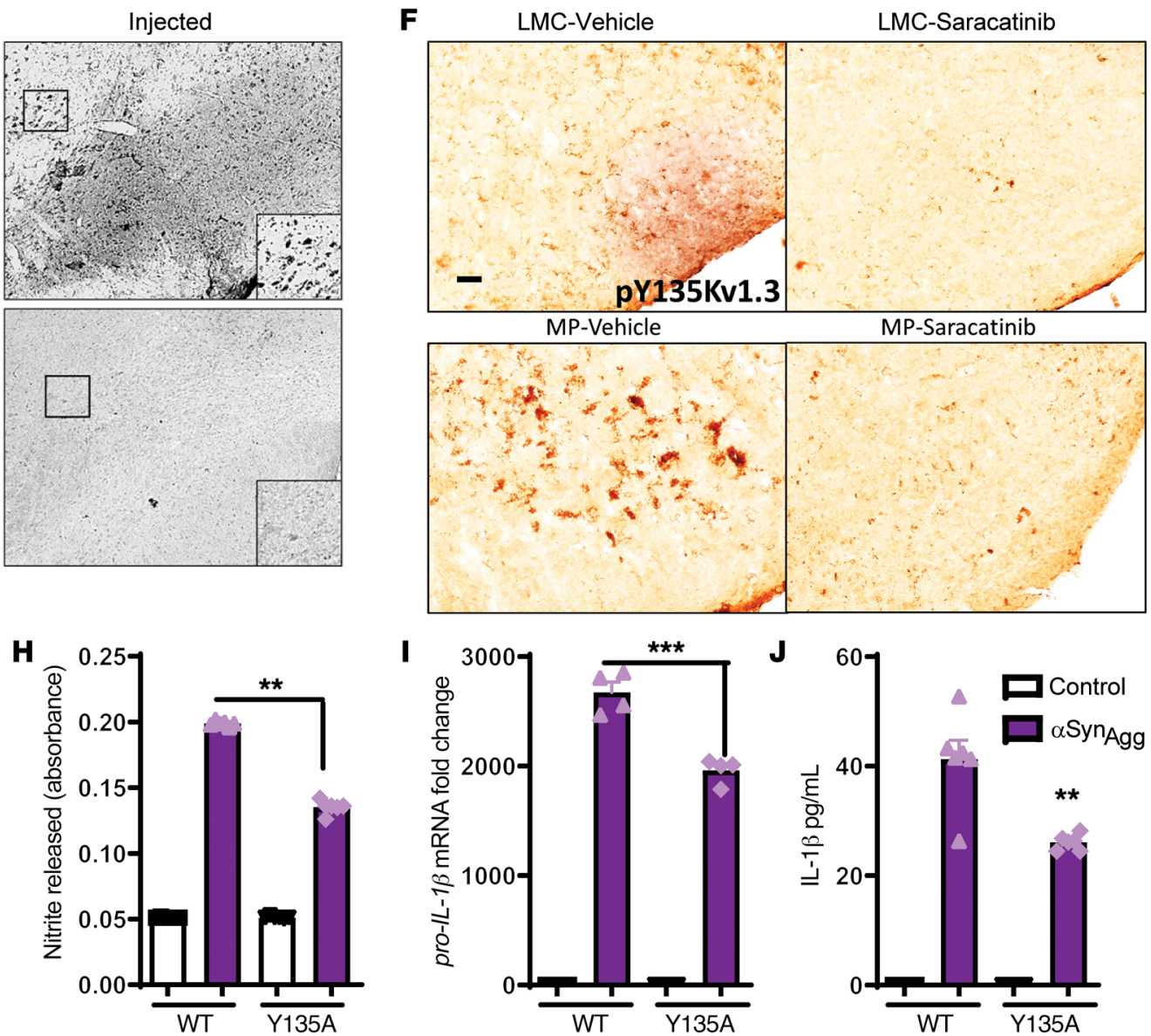

I

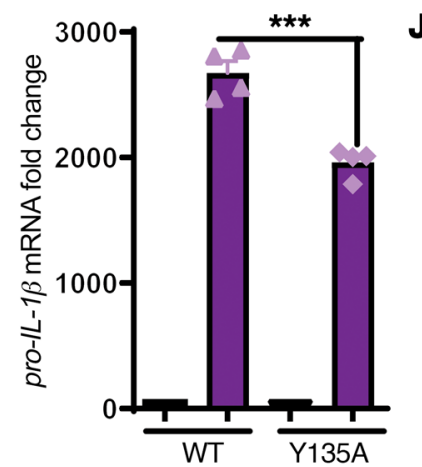

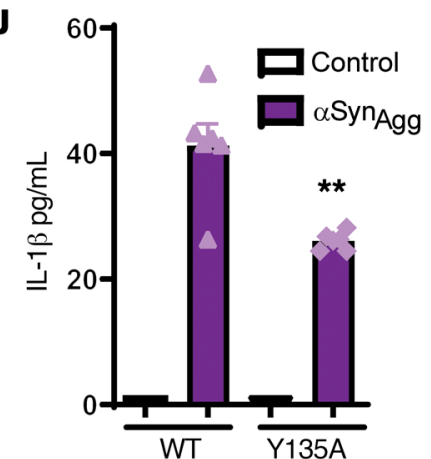

Figure 5. Fyn modulates the posttranslational modification of Kv1.3. (A) Western blot analysis of postmortem human PD and age-matched control brains showing increased phosphorylation of Kv1.3. (B) Immunoprecipitation of Fyn and Kv1.3 showing direct Fyn-Kv1.3 interaction after $\alpha S y n_{\text {Agg }}$ treatment. (C) Duolink PLA showing $\alpha \mathrm{Syn}_{\mathrm{Agg}}$-induced interaction between Kv1.3 and Fyn. Scale bar: $25 \mu \mathrm{m}$. (D) Western blot of Fyn WT and KO PMCs revealed that Kv1.3 phosphorylation at residue 135 was Fyn dependent. (E) IHC analysis of substantia nigra from Fyn ${ }^{+/+}$and Fyn ${ }^{-/-}$mice showing reduced phosphorylation of Kv1.3 after $\alpha$ Syn $_{\mathrm{PFF}}$ injection. Scale bars: $100 \mu \mathrm{m} ; 60 \mu \mathrm{m}$ (insets). (F) IHC of substantia nigra from MitoPark mice and their littermate controls showing that pharmacological inhibition of Fyn by saracatinib reduced Kv1.3 phosphorylation. Scale bar: $100 \mu \mathrm{m}$. (G-J) Immortalized MMCs were either transfected with WT Kv1.3 or aY135A Kv1.3 plasmid. (G) qRT-PCR analysis and (H) Griess assay showing reduced levels of inducible NOS (iNOS) and nitrite release, respectively, in Y135A Kv1.3-transfected cells compared with WT cells. (I) qRT-PCR analysis showing reduced IL-13 production in Y135A Kv1.3-transfected versus WT Kv1.3-transfected MMCs. (J) Luminex assay showing reduced IL-1 $\beta$ secretion in Y135A Kv1.3-transfected compared with WT Kv1.3-transfected MMCs. A 1-way ANOVA was used to compare multiple groups. In most cases, Tukey's post hoc analysis was applied. A 2-tailed Student's $t$ test was used to compare 2 groups in $\mathbf{A}$. Each dot on the bar graphs represents a biological replicate. Data are presented as the mean \pm SEM, with 3-4 biological replicates from 2-3 independent experiments. ${ }^{*} P \leq 0.05,{ }^{*} P<0.01$, and ${ }^{* *} P<0.001$. 
yses further revealed reduced $\alpha S_{\mathrm{Syg}}$-induced upregulation of Nos 2 mRNA (Figure 5G), nitrite release (Figure 5H), and the proinflammatory cytokine IL-1 $\beta$ (Figure 5, I and J). Together, these results suggest that Fyn phosphorylates Kv1.3 at residue 135 and that this Fyn-induced phosphorylation plays a role in Kv1.3-mediated inflammation in PD models.

Kv1.3 modulates neuroinflammation in a cell culture model of $P D$. To demonstrate the role of $\mathrm{Kv1} .3$ in $\alpha \mathrm{Syn}_{\mathrm{Agg}}$-induced microglial activation, we performed a series of Kv1.3-KO and overexpression studies. PMCs from Kv1.3 WT and -KO animals were cultured and treated with $\alpha \mathrm{Syn}_{\mathrm{Agg}}$ for 24 hours. Luminex assays revealed that PMCs from Kv1.3-KO mice secreted significantly less TNF- $\alpha$ (Figure 6A), IL-12 (Figure 6B), and IL-1 $\beta$ (Figure 6C) than did WT PMCs. Next, we overexpressed Kv1.3 in MMCs (45) and treated them with $\alpha \mathrm{Syn}_{\mathrm{Agg}}$ for 24 hours. Our qRT-PCR analysis revealed that Kv1.3 overexpression (Supplemental Figure 6) significantly increased $\alpha \mathrm{Syn}_{\mathrm{Agg}}$-induced mRNA levels of Nos2 (Figure 6D), pro-IL-1 $\beta$ (Figure $6 \mathrm{E}$ ), and Tnf- $\alpha$ (Figure $6 \mathrm{~F}$ ). Multiplex assays further revealed that Kv1.3 overexpression significantly induced the secretion of IL-6 (Figure 6G) and IL-12 (Figure 6H) following $\alpha \mathrm{Syn}_{\mathrm{Agg}}$ treatment. These data collectively suggest that $\mathrm{K}_{\mathrm{v}} 1.3$ plays an important role in $\alpha S_{y n} n_{\mathrm{Agg}}$-induced microglial inflammation.

The small-molecule PAP-1 is a pharmacological inhibitor that specifically blocks Kv1.3 activity (28) and is known to reduce inflammation in rodent models of autoimmune disease. We cotreated PMCs with $\alpha \mathrm{Syn}_{\mathrm{Agg}}$ and PAP-1. Patch clamping showed that PAP-1 blocked Kv1.3 currents in $\alpha \mathrm{Syn}_{\mathrm{Agg}}$-stimulated microglia (Figure 6I). A lactate dehydrogenase (LDH) assay revealed that PAP-1 blocked the $\alpha \mathrm{Syn}_{\mathrm{Agg}^{-}}$ induced release of LDH into the extracellular space (Figure 6J). PAP1 also significantly reduced the $\alpha \mathrm{Syn}_{\mathrm{Ag}}$-induced release of the proinflammatory cytokines IL-12 (Figure 6K), TNF- $\alpha$ (Figure 6L), and IL-6 (Figure 6M) as revealed by Luminex multiplex cytokine analysis. To further demonstrate the efficacy of PAP-1 in reducing microglial inflammation, MMCs were cotreated with PAP- $1(1 \mu \mathrm{M})$ and $\alpha \mathrm{Syn}_{\mathrm{Agg}}$. The Griess assay showed that PAP-1 reduced $\alpha \mathrm{Syn}_{\mathrm{Agg}}$-induced nitrite release in these immortalized microglial cells (Supplemental Figure 7A), whereas qRT-PCR analysis showed that PAP-1 reduced expression of the $\alpha \mathrm{Syn}_{\mathrm{Agg}}$-induced inflammatory genes Nos2 (Supplemental Figure 7B), $I L-6$ (Supplemental Figure 7C), TNF- $\alpha$ (Supplemental Figure 7D), and $I L-1 \beta$ (Supplemental Figure 7E). However, PAP-1 treatment did not induce the markers for alternative activation of microglia, IRF4 (Supplemental Figure 7F) and MRC1 (Supplemental Figure 7G). Luminex assays further demonstrated that PAP-1 could significantly attenuate $\alpha \mathrm{Syn}_{\mathrm{Agg}}$-induced release of the proinflammatory cytokines IL-1 $\beta$ (Supplemental Figure 7H), TNF- $\alpha$ (Supplemental Figure 7I), and IL-6 (Supplemental Figure 7J).

To advance our understanding of the role of the Kv1.3dependent signaling cascade in modulating neuroinflammation in microglia, we performed immunoblot analysis on PAP-1-treated MMCs. Western blot and ICC analyses demonstrated that PAP-1 significantly reduced $\alpha \mathrm{Syn}_{\mathrm{Agg}}$-induced NLRP3 inflammasome protein upregulation (Figure 6, $\mathrm{N}$ and $\mathrm{O}$ ). Together, these findings suggest that Kv1.3 contributes to classical microglial activation, which can be attenuated by PAP-1.

PAP-1 reduces neuroinflammation and neurodegeneration in mouse models of PD. PAP-1 and other Kv1.3 inhibitors have been widely used in animal models of inflammation including psoria- sis (46), autoimmune diabetes (47), ischemic stroke (48), and AD (26), and its chronic administration is well tolerated in mice, rats, and primates (49). To validate the therapeutic potential of PAP-1 in Parkinsonian syndromes, we used the transgenic MitoPark progressive dopaminergic neurodegenerative model of PD and the toxin-based MPTP mouse model of PD.

MitoPark mice were injected daily with $40 \mathrm{mg} / \mathrm{kg}$ (i.p.) PAP-1 from ages 14 to 20 weeks (Supplemental Figure 8A). During treatment, no significant weight loss was observed (Supplemental Figure 8B). To verify the bioavailability and brain penetrance of PAP-1, we performed liquid chromatography-mass spectrometry (LC-MS) analysis of serum and brain samples taken at the end of the study and found that total PAP-1 serum and brain concentrations in the treated animals averaged $322 \pm 122 \mathrm{nM}$ and $848 \pm 295 \mathrm{nM}$, respectively, 24 hours after the last administration (Supplemental Figure 8C). We detected no PAP-1 in the serum or brains of the vehicle-treated animals. Behavioral tests revealed that PAP-1 significantly reduced motor deficits in MitoPark mice (Figure 7, A-C). As evident from representative movement tracks (Figure 7A), PAP-1 treatment significantly increased horizontal activity in the open-field test (Figure 7B). PAP-1 also significantly increased the time spent on the rotarod by MitoPark mice (Figure 7C). Biochemical analysis of the striatum revealed that PAP-1 reduced loss of the neurotransmitter dopamine (Figure 7D) and its derivatives DOPAC (Figure 7E) and homovanillic acid (HVA) (Figure 7F) in MitoPark mice. Furthermore, stereological analysis demonstrated that PAP-1 significantly protected MitoPark mice from the loss of dopaminergic neurons (Figure 7G). Fluoro-Jade and IHC staining further revealed that PAP-1 reduced the extent of microgliosis and neuronal degeneration in MitoPark mice (Supplemental Figure 8D).

Next, we evaluated the efficacy of PAP-1 in protecting against MPTP-induced neuronal loss and inflammation (Supplemental Figure 9A). As expected, the LC-MS analysis revealed that PAP1 crossed the blood-brain barrier (Supplemental Figure 9B). IHC analysis demonstrated that PAP-1 reduced MPTP-induced microgliosis in both the striatum and SN (Figure $7 \mathrm{H})$. Skeletonization analysis (50) revealed that PAP-1 reduced microglial soma size and increased the number of branches compared with MPTP treatment alone (Figure 7I). Increased microglial soma size and decreased branch numbers are indicative of classical activation of microglia. Moreover, qRT-PCR analysis revealed that Kv1.3 inhibition reduced MPTP-induced expression of the proinflammatory cytokines TNF- $\alpha$ and pro-IL-1 $\beta$ (Figure 7J). Biochemical analysis showed that PAP-1 significantly increased the amount of dopamine (Supplemental Figure 9C) and its metabolites DOPAC (Supplemental Figure 9D) and HVA (Supplemental Figure 9E) compared with MPTP treatment alone. TH neuronal staining and stereological analysis further demonstrated that PAP-1 protected against MPTP-induced dopaminergic neuronal loss in the SN (Figure 7, $\mathrm{K}$ and L).

Finally, we also used the $\alpha \mathrm{Syn}_{\mathrm{PFF}}$ mouse model to further demonstrate the effect of Kv1.3 inhibition as a potential therapeutic target for PD. We injected $\alpha \mathrm{Syn}_{\mathrm{PFF}}$ into the striatum, and 4 months after injection, PAP-1 $(40 \mathrm{mg} / \mathrm{kg})$ was administered for 30 days (Figure 8A). As evident from representative movement tracks (Figure 8B), PAP-1 significantly decreased the rest time (Figure 8C) and increased horizontal activity (Figure $8 \mathrm{D}$ ) and total distance 

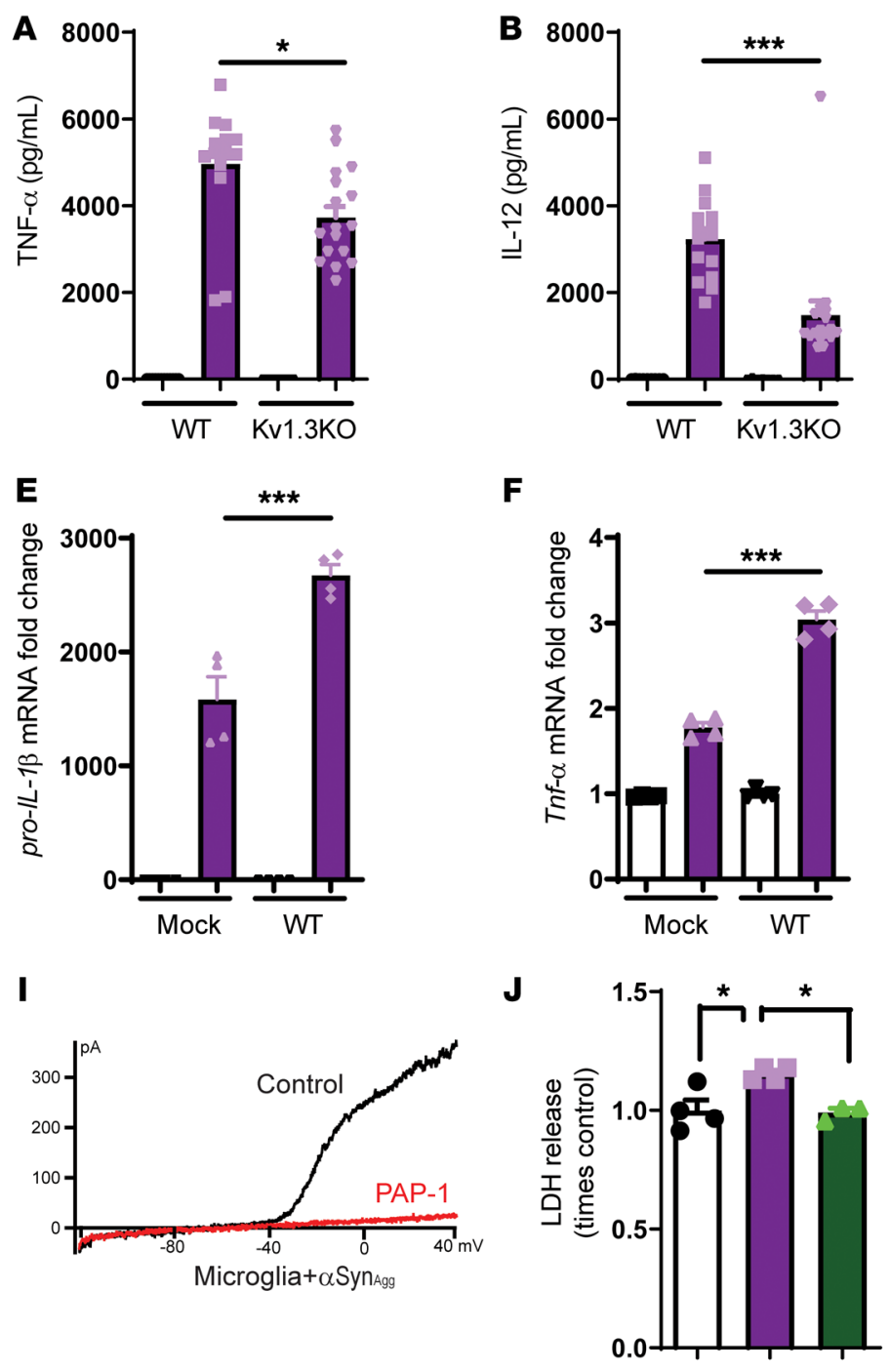

M
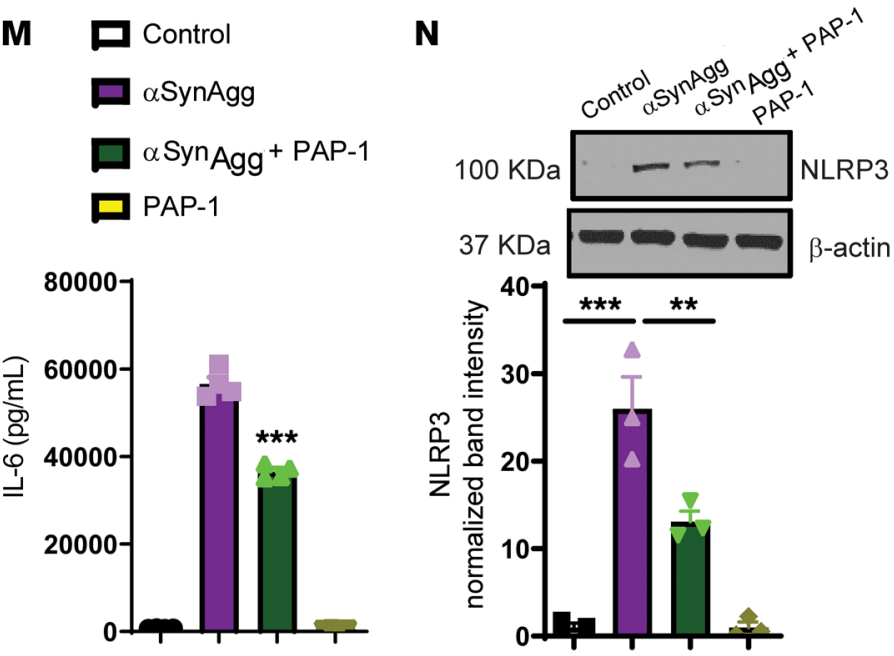

$\mathbf{F}$

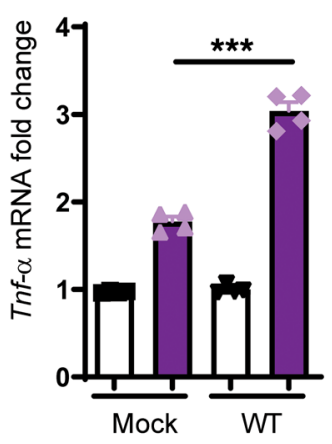

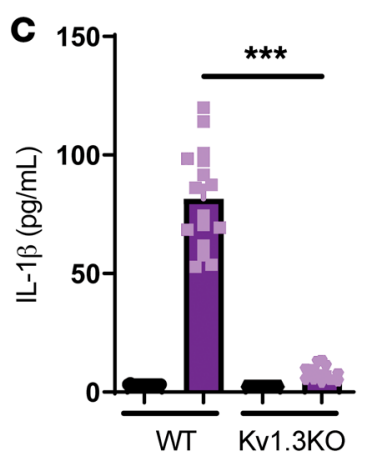

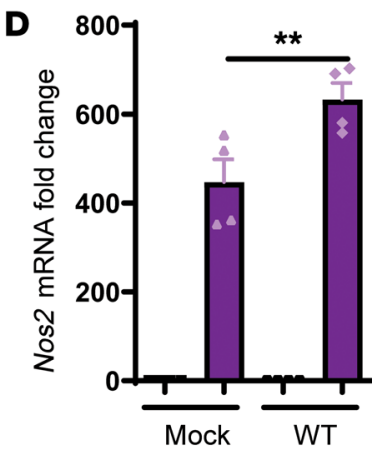

G

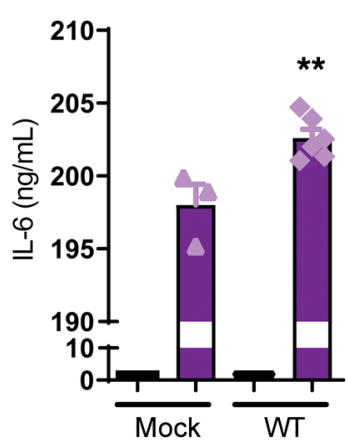

H

$\mathbf{K}$
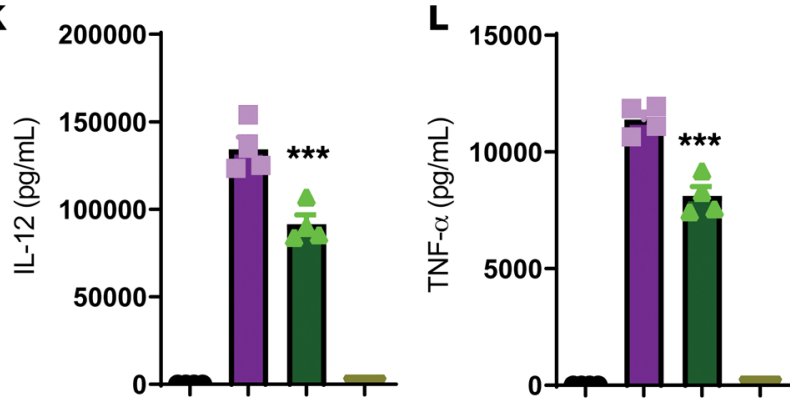

0
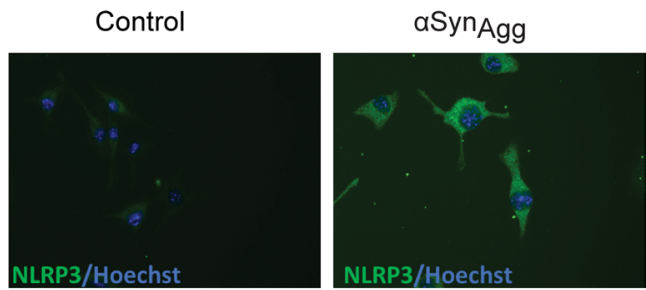

$a_{\text {Syn }}$ Agg + PAP-1

PAP-1
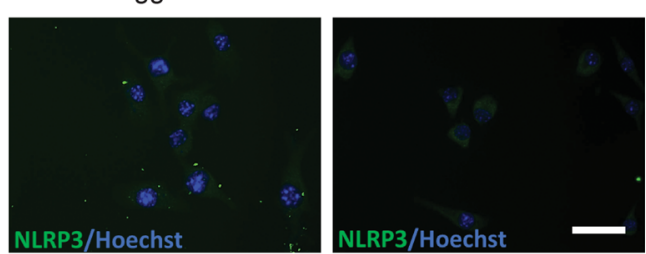
Figure 6. Kv1.3 modulates neuroinflammation in a cell culture model of PD. (A-C) Kv1.3 WT and KO PMCs were treated with $1 \mu \mathrm{M} \alpha \mathrm{Syn}_{\text {Agg }}$ for 24 hours. Luminex analysis shows that Kv1.3 $\mathrm{KO}$ reduced the release of the $\alpha$ Syn $_{\mathrm{Agg}}$-induced proinflammatory factors (A) TNF- $\alpha$, (B) IL-12, and (C) IL-1 $1 \beta$. (D-H) Immortalized MMCs were transfected with WT a Kv1.3 plasmid, and then 48 hours after transfection, cells were treated with $1 \mu \mathrm{M} \alpha \mathrm{Syn}_{\mathrm{Agg}}$ for 24 hours. (D-F) qRT-PCR analysis showing that Kv1.3 overexpression aggravated $\alpha \mathrm{Syn}_{\mathrm{Agg}}$-induced production of the proinflammatory factors (D) Nos2, (E) pro-IL-1 $\beta$, and (F) TNF- $\alpha$. (G and $\mathbf{H}$ ) Luminex analysis showing that Kv1.3 overexpression potentiated the release of the proinflammatory factors (C) IL-6 and (H) IL-12. (I) Voltage ramp from $-120 \mathrm{mV}$ to $40 \mathrm{mV}$ elicited a characteristic outward rectifying current in $\alpha \mathrm{Syn}_{\mathrm{Agg}}$-treated microglia that was sensitive to the Kv1.3-selective inhibitor PAP-1. (J) LDH assay showing that PAP-1 reduced $\alpha \mathrm{Syn}_{\mathrm{Agg}}$-induced $\mathrm{LDH}$ release from microglial cells. (K-M) Luminex assay revealing that PAP-1 attenuated the $\alpha S_{y n} n_{\text {Agg }}$-induced proinflammatory factors (K) IL-12, (L) TNF- $\alpha$, and (M) IL-6. (N) Western blot analysis demonstrating that PAP-1 reduced $\alpha \mathrm{Syn}_{\mathrm{Agg}}$-induced NLRP3 expression. (0) ICC analysis revealed that PAP-1 reduced NLRP3 expression induced by $\alpha \mathrm{Syn}_{\mathrm{Agg}}$. Scale bar: $25 \mu \mathrm{m}$. A 1-way ANOVA was performed to compare multiple groups. In most cases, Tukey's post hoc analysis was applied. Each dot on the bar graphs represents a biological replicate. Data are presented as the mean \pm SEM, with 3-4 biological replicates from 2-3 independent experiments. ${ }^{*} P \leq 0.05$, ${ }^{* *} P<0.01$, and ${ }^{* *} P<0.001$.

traveled (Figure 8E). Furthermore, biochemical analysis revealed that PAP-1 restored the $\alpha \mathrm{Syn}_{\mathrm{PFF}}$-induced loss of dopamine (Figure 8F) and its metabolite DOPAC (Figure 8G). Western blot analysis further confirmed that PAP- 1 reduced the $\alpha \mathrm{Syn}_{\mathrm{PFF}}$-induced loss of $\mathrm{TH}$ expression in the SNpc region of the brain (Figure $8 \mathrm{H}$ ). Collectively, these preclinical studies suggest that PAP-1 can reduce microgliosis, inflammation, and neuronal loss in PD models and that inhibition of Kv1.3 by a brain-penetrant small molecule has clinical potential for PD treatment.

\section{Discussion}

Although neuroinflammation is one of the key pathophysiological hallmarks of neurodegenerative disorders, including PD, the molecular mechanisms and the key regulators of this chronic, sustained inflammation are not well understood (4). Recently, various antiinflammatory drugs targeting cellular pathways modulating inflammation have gained importance in clinical and preclinical trials in $\mathrm{AD}$ and $\mathrm{PD}(51,52)$. In the present study, we demonstrate for the first time to our knowledge that the voltage-gated potassium channel Kv1.3 is upregulated in microglia in Parkinsonism. We showed previously that exposure to an inflammagen activates the Src family kinase Fyn, which regulates the p38 MAPK and the NF- $\kappa$ B pathways in microglial cells (27) and that Fyn phosphorylates PKC $\delta$, a novel PKC isoform that also regulates the microglial NF- $\mathrm{B}$ pathway (38). In this study, we reveal that both the p38 MAPK and the classical NF- $\mathrm{BB}$ pathways mediate the transcriptional regulation of Kv1.3. Furthermore, we identify the Fyn/PKC $\delta$ inflammatory pathway as a major regulator of Kv1.3 transcription in response to inflammatory stimuli. We demonstrate that the phosphorylation profile of Kv1.3 can be modulated by $\alpha \mathrm{Syn}_{\mathrm{Agg}}$ and that Fyn kinase plays an important role in this $\alpha \mathrm{Syn}_{\mathrm{Agg}}$-induced Kv1.3 phosphorylation. Finally, we show that PAP-1, a brain-penetrant, small-molecule Kv1.3 inhibitor, can reduce neuroinflammation and neurodegeneration in multiple animal models of PD, demonstrating the translational relevance of our study.
The role of the microglial neuroinflammatory response in the neurodegenerative process of PD was first identified over 3 decades ago (53), but the exact molecular mechanism underlying the sustained inflammation remains unresolved. Meta-analyses have revealed that NSAIDs can protect against neurodegenerative disorders (54), suggesting that an effective translational strategy to dampen neuroinflammation could offer neuroprotection in PD. Recent studies have shown that $\alpha \mathrm{Syn}_{\mathrm{Agg}}$, a major component of Lewy bodies and neurites, can be transferred from cell to cell in a prion-like manner, leading to the spread of disease pathology $(55,56)$. Although $\alpha \operatorname{Syn}_{\mathrm{Agg}}$ can induce inflammation in cell cultures and animal models of PD, the downstream signaling mechanism is not well understood (38). In this study, we show that the microglial Kv1.3 potassium channel was highly upregulated in response to $\alpha \mathrm{Syn}_{\mathrm{Agg}}$. Interestingly, we show that surface Kv1.3 expression levels (Figure 1E) increased to a much greater degree than whole-cell protein levels (Figure 1C), suggesting that the trafficking of Kv1.3 to the surface plays an important role in this upregulation. Previous studies have reported that adaptor protein 1 (AP-1), which is responsible for clathrin-coated vesicle formation, governs Kv1.3 trafficking and that Fyn plays an important role in governing AP-1 expression $(57,58)$. Therefore, future studies looking into the role of the Fyn/ $\mathrm{AP}-1$ axis in $\alpha \mathrm{Syn}_{\mathrm{Agg}}$-induced Kv1.3 trafficking are warranted. Currently, the exact reason for the difference in magnitude between Kv1.3 mRNA and protein upregulation is not entirely clear (Figure 1 , B versus C), but it probably results from changes in Kv1.3 mRNA turnover, translational control, or the protein turnover rate. The difference could also be due to the higher sensitivity of a qRT-PCRbased mRNA assay compared with the semiquantitative Western blot method. We observed the induction of Kv1.3 in multiple in vivo models of PD, recapitulating various key hallmarks of PD. Moreover, we found that Kv1.3 was also upregulated in patients with Parkinsonian syndrome (PD and DLB), demonstrating the clinical relevance of our findings.

Ion channels play an important role in the immune system by regulating membrane potential and calcium signaling. The potassium channel Kv1.3, in particular, is widely regarded as a promising target for immunosuppression (59) and has been demonstrated to constitute a valid target for treating $\mathrm{T}$ cell-mediated autoimmune diseases in animal models of multiple sclerosis, autoimmune diabetes, rheumatoid arthritis, and psoriasis $(47,59)$. Kv1.3 blockers have also been shown to rescue neurogenesis by inhibiting granzyme B-releasing $\mathrm{CD}^{+} \mathrm{T}$ cells $(60)$. In microglial cells, the resident immune cells of the brain, Fordyce et al. (22) demonstrated that Kv1.3 is involved in microglia-mediated neuronal killing, whereas others (61) have implicated Kv1.3 in HIV-induced microglial neurotoxicity. More recently, 2 studies have shown that increased Kv1.3 expression in microglia is associated with classical "M1-like" activation (23) and that Kv1.3 is involved in modulating microglia-induced inflammation, phagocytosis, and antigen presentation (62). In humans, increased microglial Kv1.3 expression has further been demonstrated in the postmortem brains of $\mathrm{AD}$ (25) and stroke (48) patients. Interestingly, the Kv1.3 blocker PAP-1 has been found to reduce neuroinflammation and improve cognition or neurological deficits in rodent models of $\mathrm{AD}$ and ischemic stroke $(26,48)$. However, Kv1.3-mediated current was reportedly reduced in striatal cholinergic neurons in a PD mouse model (63), similar to the finding that ion channel activity decreases 
A

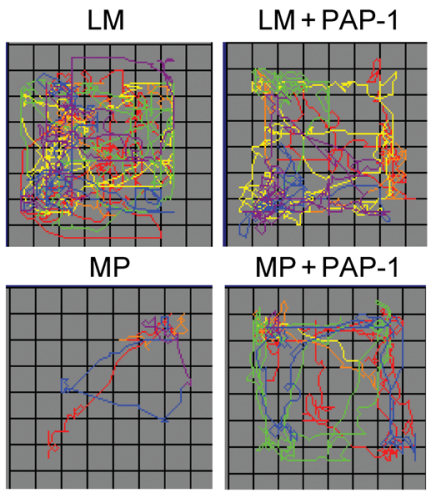

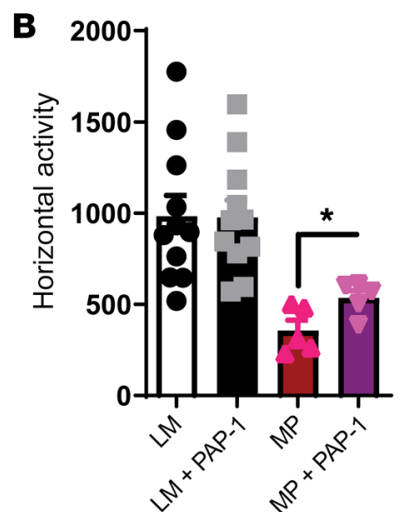
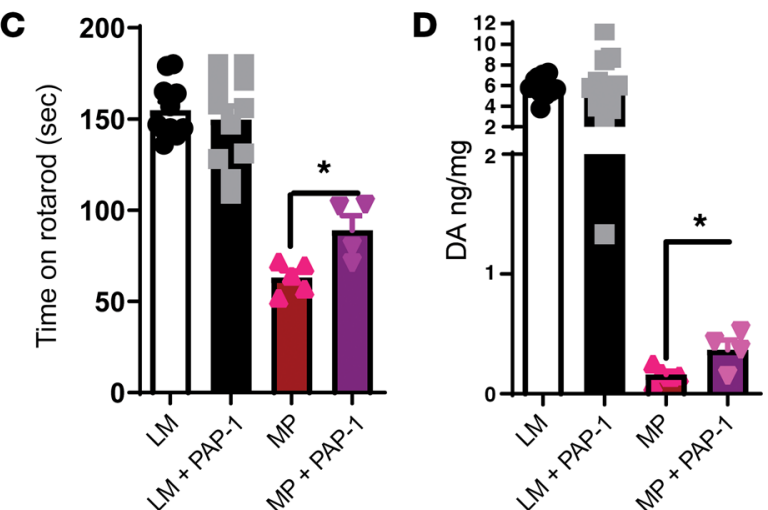
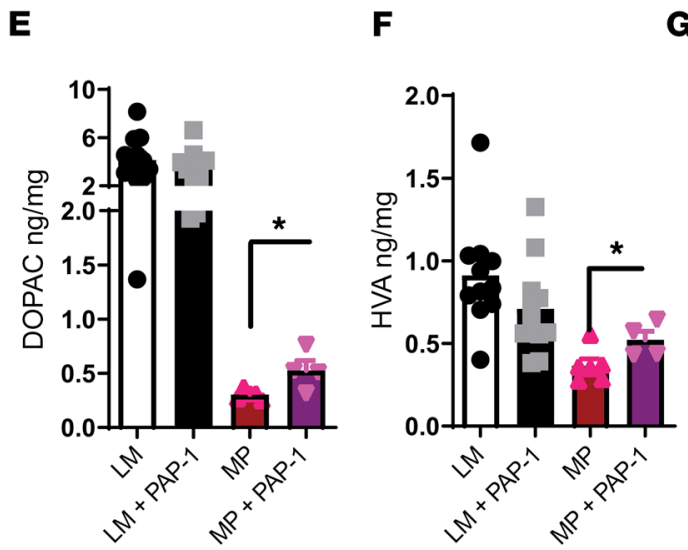

H
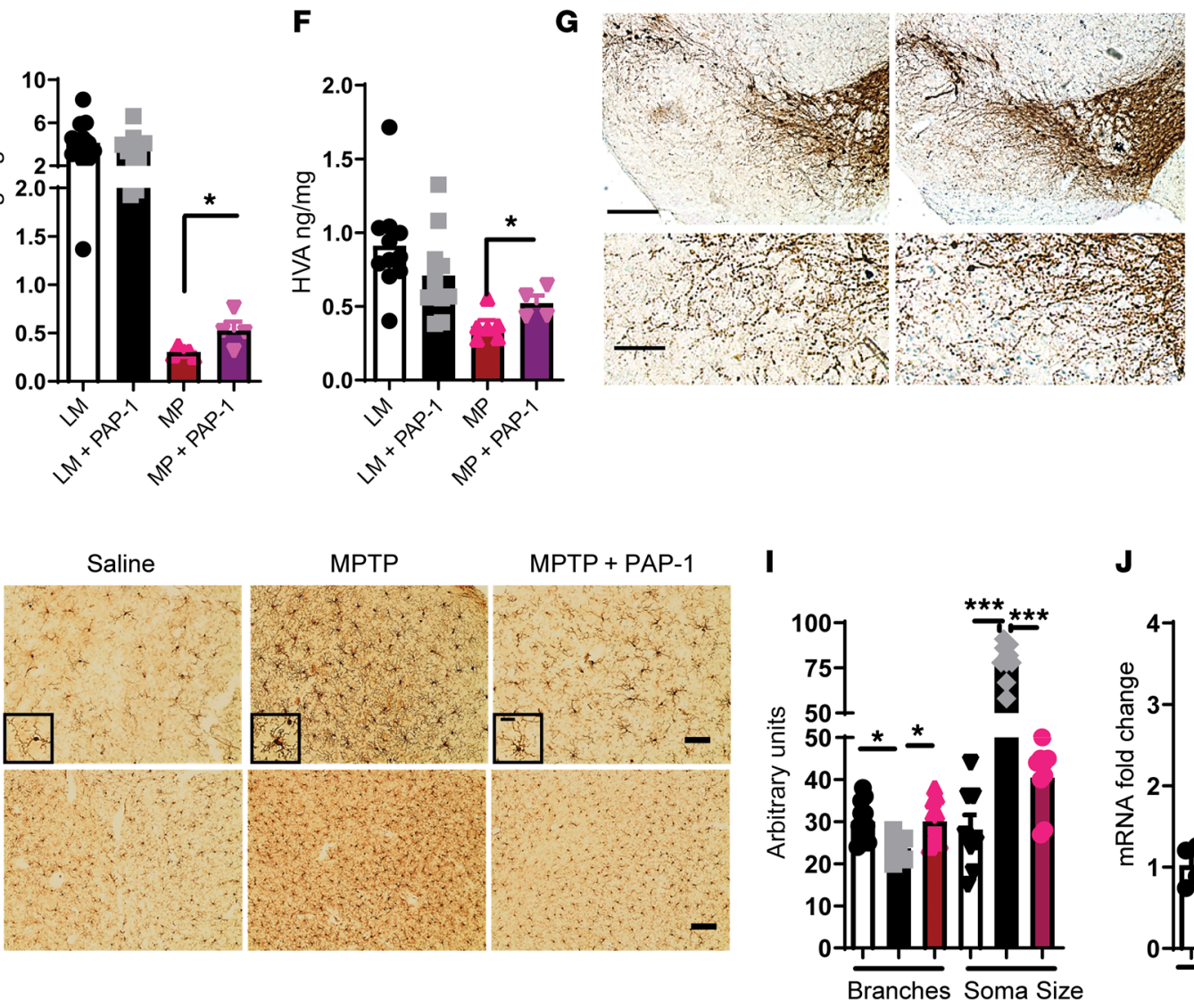
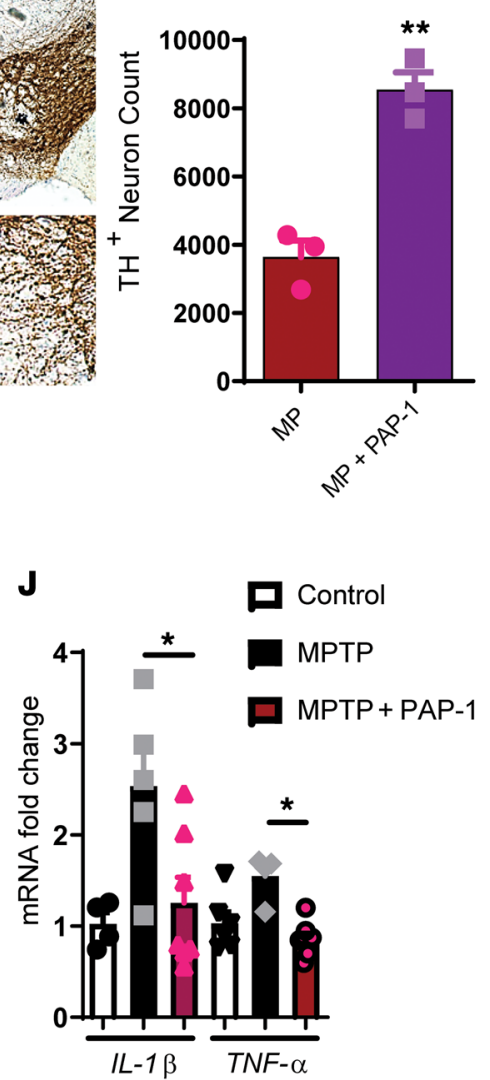

K $2 x$

Saline
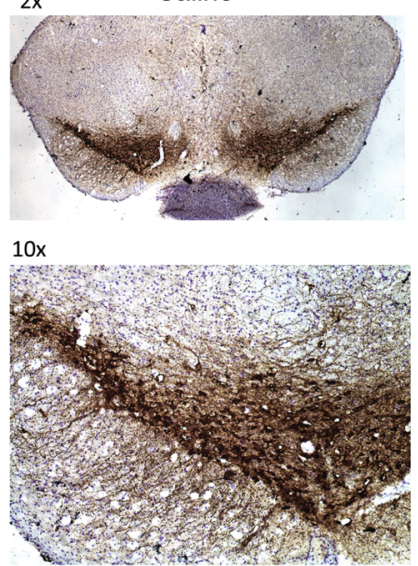

MPTP
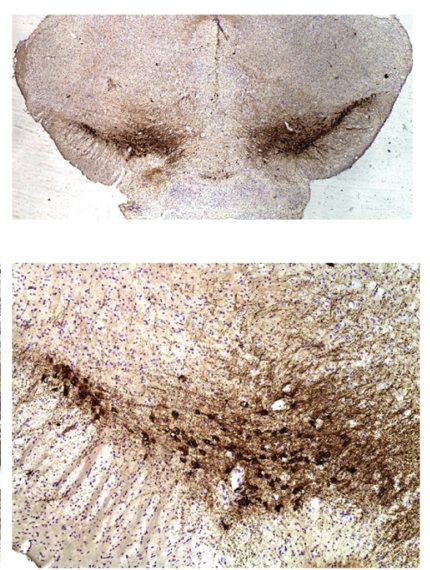
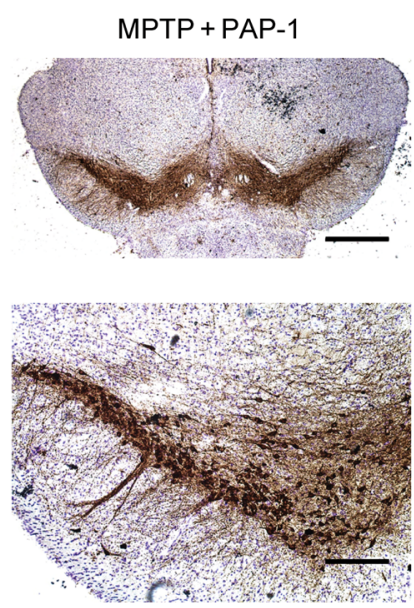

L

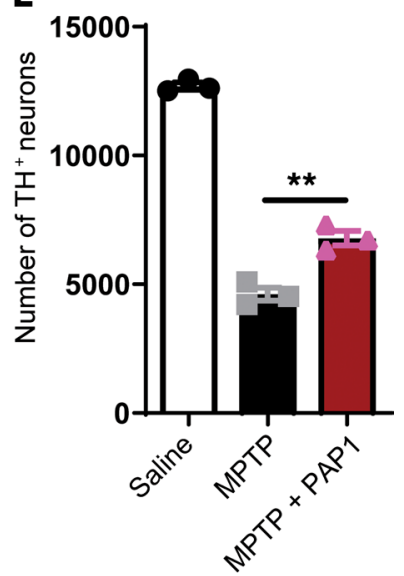


Figure 7. PAP-1 reduces inflammation and neurodegeneration in mouse models of PD. (A-H) MitoPark mouse model. (A) Representative movement tracks showing that PAP-1 rescued movement deficits of MitoPark mice at 20 weeks. (B) VersaMax open-field test showed increased horizontal activity of MitoPark mice treated with PAP-1 compared with the vehicle-treated control group. (C) Behavior test revealed increased time spent on the rotarod by MitoPark mice treated with PAP-1 compared with the vehicle-treated group. (D-F) HPLC showing that PAP-1 treatment protected MitoPark mice from loss of (D) dopamine (DA), (E) DOPAC, and (F) HVA. (G) IHC of SNpc showing that PAP-1 protected against loss of TH-positive neurons in MitoPark mice and stereology analysis of the SNpc showing that PAP-1 decreased the loss of TH-positive neurons in MitoPark mice. Scale bars: $200 \mu \mathrm{m}$ (top panel); $100 \mu \mathrm{m}$ (bottom panel). (H-L) MPTP mouse model. (H) IHC of MPTP-exposed substantia nigra and striatum showing that PAP-1 altered microgliosis. Scale bars: $50 \mu \mathrm{m}$ (top panel); 20 $\mu \mathrm{m}$ (insets); $100 \mu \mathrm{m}$ (bottom panel). (I) ImageJ analysis of MPTP-exposed substantia nigra showing that PAP-1 reduced soma size and increased the number of microglial branches. (J) qRT-PCR analysis of striatum after MPTP showing reduced mRNA expression of proinflammatory factors IL-1 $\beta$ and TNF- $\alpha$. (K) IHC of SNpc showing that PAP-1 protected against MPTP-induced loss of TH-positive neurons. Scale bars: $500 \mu \mathrm{m}$ (top panel); $200 \mu \mathrm{m}$ (bottom panel). (L) Stereological analysis of the SNpc showing that PAP-1 decreased the loss of TH-positive neurons after MPTP treatment. One-way ANOVA was used to compare multiple groups. In most cases, Tukey's post hoc analysis was applied. Each dot on the bar graphs represents a biological replicate. Data are presented as the mean \pm SEM, with 3-7 animals per group. ${ }^{*} P \leq 0.05,{ }^{* *} P<0.01$, and ${ }^{* * *} P<0.001$.

in dopaminergic neurons in animal models of PD (64). It is not clear how these findings of PD-dampened ion channel activity relate to the proof-of-concept animal studies reported here, which show that inhibition of microglial Kv1.3 with PAP-1 protected against neuronal loss and inflammation in PD models and which further support an important role for Kv1.3 in neuroinflammation.

The mechanistic underpinnings of Kv1.3 transcriptional regulation remain largely unknown. Recent Kv1.3 studies have implicated the enzyme cereblon in its epigenetic regulation (35), its age-dependent methylation in patients with colorectal cancer (65), and in DNA methylation of its promoter in breast cancer (66). Although p38 MAPK in microglia has been shown to modulate Kv1.3 expression in HIV glycoprotein 120-induced neurotoxicity (61), the proximal signaling node that upregulates Kv1.3 has yet to be defined. In this study, we demonstrated that the NF- $\kappa B$ and $\mathrm{p} 38$ MAPK pathways contribute to the $\alpha \mathrm{Syn}_{\mathrm{Agg}}$-induced transcriptional upregulation of Kv1.3. Our group recently showed that Fyn is induced in cell culture and animal models of PD and is important in modulating microglial inflammation by mediating the NF- $\kappa \mathrm{B}$ and p38 MAPK pathways (10, 27). Fyn inhibition has been shown to reduce neuroinflammation in $\mathrm{AD}$ (52). We also showed that Fyn is upstream of the proapoptotic and proinflammatory kinase $\operatorname{PKC} \delta(27,38)$. Fyn also has a role in regulating inflammation in $\mathrm{AD}$, whereby inhibition of Fyn kinase with saracatinib was found to protect against neurodegeneration in rodent models of AD (52). We report here that Fyn and PKC $\delta$ regulate Kv1.3 transcription by regulating NF- $\mathrm{\kappa B}$ and p38 MAPK signaling. We induced a significant reduction in Kv1.3 expression as well as in its transcriptional upregulation by pharmacologically inhibiting Fyn or genetically ablating Fyn expression. Collectively, our results identify, for the first time to our knowledge, the Fyn/PKC $\delta$ axis as a key upstream signaling node in modulating Kv1.3 transcriptional upregulation.
Posttranslational modification of potassium channels leading to modulation of channel activity has been actively researched. Kv1.3 has multiple phosphorylation sites, including both tyrosine and serine/threonine $(39,40)$. Jimenez-Perez et al. (67) implicated the tyrosine phosphorylation sites of Kv1.3 in modulating proliferation. Holmes et al. (39) demonstrated that endogenous kinases and phosphatases continuously phosphorylate and dephosphorylate Kv1.3 and that any change in this dynamic phosphorylation/dephosphorylation can drastically alter Kv1.3 activity. Various tyrosine kinase proteins, including $\mathrm{v}$-Src, are known to phosphorylate Kv1.3, and different adaptor proteins, including Grb10, modulate this phosphorylation of Kv1.3, thereby differentially regulating its activity $(21,42)$. We show here that Fyn, a nonreceptor tyrosine kinase, can phosphorylate Kv1.3, thereby modulating its function. Though we have shown a direct interaction between Fyn and Kv1.3, our Kv1.3 Tyr135 mutant transfection study revealed a modest effect on inflammation. This may be due to other possible Fyn phosphorylation sites on Kv1.3. Further studies are required to fully understand the role of Fyn in modulating Kv1.3 activity.

Collectively, we demonstrate that Kv1.3 is upregulated in Parkinsonian models. This upregulation was proximally controlled by the Fyn/PKC $\delta$ kinase signaling cascade through the downstream mediator of the NF- $\mathrm{BB}$ and p38 MAPK pathways. Fyn could further phosphorylate Kv1.3, which modulated Kv1.3 channel activity. We show that Kv1.3 upregulation in PD models plays a major role in neuroinflammation-mediated neurodegeneration. These findings further demonstrate a probable Kv1.3-mediated signaling cascade (Supplemental Figure 10) that can modulate microglial inflammation in Parkinsonism. Our comprehensive preclinical evaluation of the Kv1.3 blocker PAP-1 in 3 different animal models of PD, including MitoPark transgenic, MPTP, and $\alpha \mathrm{Syn}_{\mathrm{PFF}}$ mouse models, provides credence to the translational relevance of Kv1.3 modulation in PD. The diverse roles of Kv1.3 emerging from our findings, together with other studies, reinforce the potential therapeutic implications of Kv1.3 modulators.

\section{Methods}

Further information can be found in Supplemental Methods.

Cell culture, primary culture, and treatments. Primary mouse microglia (PMCs) were isolated from mixed glial cultures using a magnetic bead separation protocol published previously by our laboratory $(68,69)$. The mixed glia cultures from P0 mouse pups from C57BL/6 J mice were prepared. Primary cells were maintained in DMEM-F12 supplemented with $10 \%$ FBS, $1 \%$ sodium pyruvate, $1 \%$ glutamine, $1 \%$ penicillin-streptomycin, and $1 \%$ nonessential amino acids. An immortalized MMC line was donated by D.T. Golenbock (University of Massachusetts Medical School, Worcester, Massachusetts, USA). The MMC line was characterized by our group $(45,70-72)$ and Halle et al. (73) and cultured in DMEM medium containing 10\% FBS, 1\% glutamine, and $1 \%$ penicillin-streptomycin. Treatments were performed in $2 \%$ FBS-containing medium. GFP and human $\alpha$ Syn-overexpressing AAVs (AAV-GFP, $0.95 \times 10^{13}$ viral particles/mL and AAV-SYN, $1 \times 10^{13}$ viral particles $/ \mathrm{mL}$ ) were obtained from the University of North Carolina Viral Vector Core (Chapel Hill, North Carolina, USA). Both viral vectors were of the AAV- 5 serotype and coded for GFP and human $\alpha$ Syn, respectively, under a chicken $\beta$-actin promoter. 
A

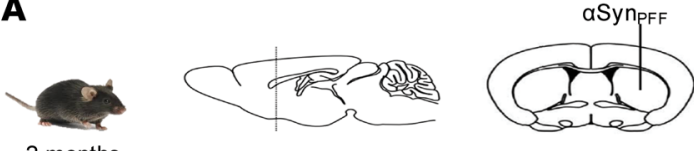

6 months

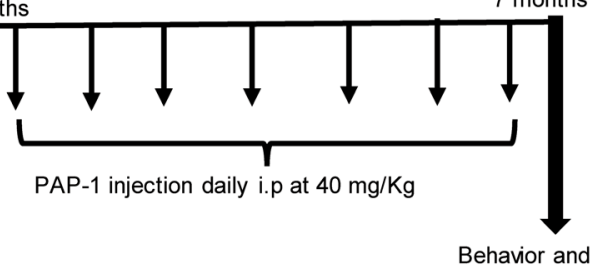

B

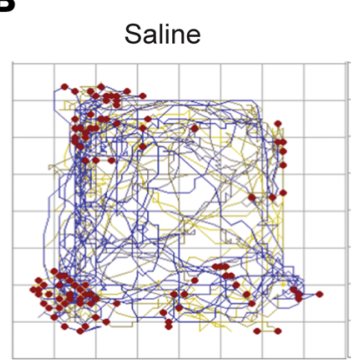

E

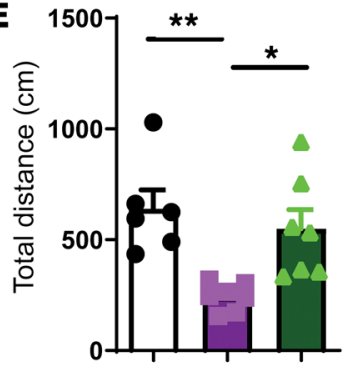

F

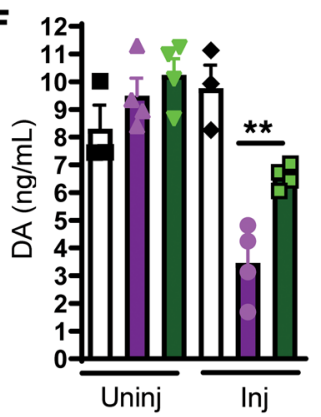

aSynPFF+PAP-1

aSynpFF

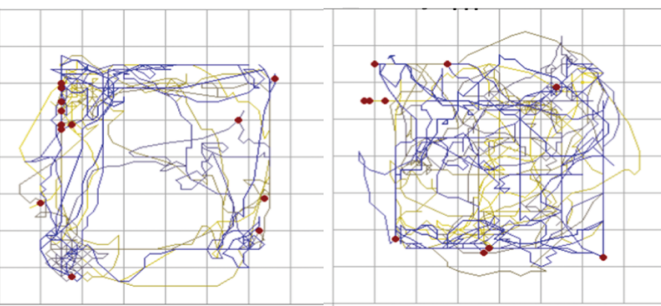

C

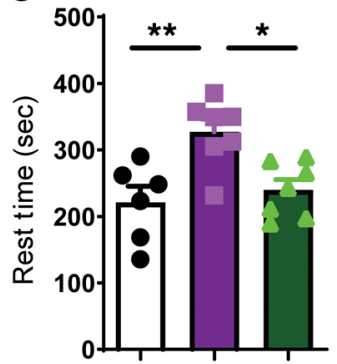

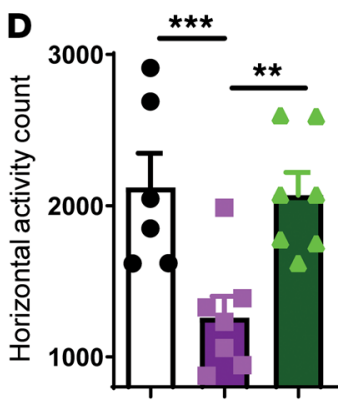

G

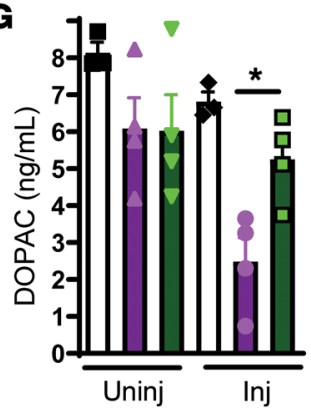

Saline

एSyn PFF

$\propto$ Syn $_{\mathrm{PFF}}+\mathrm{PAP}-1$

H

Inj Uninj Inj Uninj Inj Uninj

\begin{tabular}{|c|c|c|c|c|c|c|}
\hline$\alpha$ Syn $_{\mathrm{PFF}}$ & - & - & + & + & + & + \\
\hline PAP-1 & - & - & - & - & + & + \\
\hline
\end{tabular}

$50 \mathrm{kDa}$

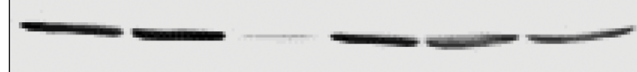

$\mathrm{TH}$

$37 \mathrm{kDa}$

$\beta$-actin

Figure 8. Kv1.3 inhibition protects against $\alpha S_{\mathrm{Sy}} \mathrm{n}_{\mathrm{PFF}}$-induced behavior deficit and dopaminergic neuronal loss. (A) Treatment paradigm corresponding to the $\alpha$ Syn $_{\mathrm{PFF}}$ mouse model of PD. (B) Representative movement tracks showing that PAP-1 rescued movement deficits induced by $\alpha \mathrm{Syn}_{\mathrm{PFF}}$. (C-E) A VersaMax open-

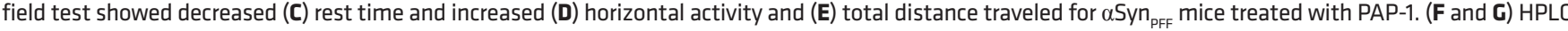
showing that PAP-1 treatment protected against loss of (F) dopamine and (G) DOPAC induced by $\alpha$ Syn $_{\mathrm{PFF}}$. $(\mathbf{H})$ Western blot analysis of TH showing loss of TH induced by $\alpha \mathrm{Syn}_{\mathrm{PFF}}$ in the SNpc region. A 1-way ANOVA was used to compare multiple groups. In most cases, Tukey's post hoc analysis was applied. Each dot on the bar graphs represents a biological replicate. Data are presented as the mean $\pm \mathrm{SEM}$, with $3-7$ animals per group. ${ }^{*} P \leq 0.05$, ${ }^{* *} P<0.01$, and ${ }^{* * *} P<0.001$.

Immortalized B cells from patients with PD and age-matched controls were obtained from the Coriell Institute for Medical Research (CIMR). Cell cultures of lymphoblasts obtained from the CIMR were maintained in RPMI 1640 media supplemented with $15 \%$ FBS, 2 mM L-glutamine, and 1\% penicillin/streptomycin. The suspension cultures were kept in upright vented-cap T25 flasks with $10 \mathrm{~mL}$ media in incubators maintained at $37^{\circ} \mathrm{C}$ and $5 \% \mathrm{CO}_{2}$. Cells were ini- tially seeded at 500,000 viable cells $/ \mathrm{mL}$ and subcultured when they reached 1,000,000 viable cells/mL. Primary human microglial cells were a gift from Douglas Walker (Banner Sun Health Research Institute, Sun City, Arizona, USA).

Animal studies. All animals were housed under standard conditions of constant temperature $\left(22 \pm 1^{\circ} \mathrm{C}\right)$, humidity (relative, $\left.30 \%\right)$, and a 12-hour light/12-hour dark cycle. 
Human samples. SN tissues from patients with PD and agematched controls were obtained from the Miami Brain Endowment Bank at the University of Miami (Miami, Florida, USA). The patients with PD were $82 \pm 7.65$ years of age, and the unaffected controls were $80 \pm 7.85$ years of age. The samples were run and analyzed blindly.

Transfection study. The Kv1.3 WT and mutant plasmids were provided by Debra A. Fadool of Florida State University (Tallahassee, Florida, USA). For transfection, 250,000 MMCs were plated in $2 \mathrm{~mL}$ media per well on a 6-well plate. After 24 hours, cells were transfected with $3 \mu \mathrm{g}$ plasmid DNA using Lipofectamine 2000 (Thermo Fisher Scientific) according to the manufacturer's protocol. The media were changed 12 hours after transfection and treated 48 hours after transfection.

Western blot analysis. Immunoblot analyses were performed following protocols described in our previously published studies $(27,38)$. Briefly, cells or tissues were homogenized and lysed using modified RIPA buffer. Proteins were normalized using a Bradford assay before loading onto a SDS-acrylamide gel. Protein (10-40 $\mu \mathrm{g})$ was loaded into each well of $10 \%-18 \%$ acrylamide gels, which were run at $110 \mathrm{~V}$ for 2-3 hours at $4^{\circ} \mathrm{C}$. Following separation, the proteins were transferred onto nitrocellulose membranes at $27 \mathrm{~V}$ for 18 hours at $4^{\circ} \mathrm{C}$. After transfer, the membranes were blocked with LI-COR blocking buffer for 45 minutes and incubated in primary and then secondary antibodies following the manufacturer's protocol. The primary antibodies included anti-Kv1.3 (Alomone Labs, 1:1000) (Research Resource Identifier [RRID]: AB_2040151), anti-Kv1.3 (MilliporeSigma 1:1000) (RRID: AB_2265087), anti-p-p38 (Cell Signaling Technology, 1:1000) (RRID: AB_331641), anti-p38 (Cell Signaling Technology, 1:1000) (RRID: AB_330713), anti-p-Kv1.3 (MilliporeSigma, 1:1000, catalog SAB4504254), anti-PKC $\delta$ (Santa Cruz Biotechnology, 1:500) (RRID: AB_628145), anti-NLRP3 (AdipoGen, 1:1000) (RRID: AB_2490202), and anti-active MAPK (Promega, 1:2000). The secondary antibodies included IR-800-conjugated goat anti-mouse IgG (LI-COR, 1:20000) and IR-700-conjugated goat anti-rabbit IgG (LI-COR 1:20,000).

$q R T-P C R$. For RNA isolation, we followed previously published protocols $(70,74)$. Briefly, TRIzol Reagent (Thermo Fisher Scientific) was used to isolate total RNAs from cells or tissues. A NanoDrop spectrophotometer (Thermo Fisher Scientific) was used to quantify RNA, and $1 \mu \mathrm{g}$ RNA was converted to cDNA using the High Capacity cDNA Synthesis Kit from Applied Biosystems (catalog 4368814). A quantitative SYBR Green PCR assay was performed using $\mathrm{RT}^{2} \mathrm{qPCR}$ SYBR Green Master Mix (Agilent Technologies) and prevalidated primers. The following validated primers from QIAGEN were used: pro-IL-1 $(\mathrm{QT01048355),} \mathrm{Nos2} \mathrm{(QT00100275),} \mathrm{Mfn2} \mathrm{(QT00134295),}$ CSF2 (QT00251286), IL-6 (QT00098875), TNF- $\alpha$ (QT00104006), mouse Kv1.3 (QT00257467), human Kv1.3 (QT00211197), Kv1.1 (QT01537263), Kv1.5 (QT00268387), KCa3.1 (QT00105672), Fyn (QT00176666), and 18S rRNA (QT02448075). The primers for MRC1 and IRF4 were synthesized at ISU's DNA facility. The fold change in gene expression was determined by the $\Delta \Delta \mathrm{Ct}$ method, where Ct was the threshold value. 18S rRNA was used as the housekeeping gene.

IHC and ICC. IHC was performed on 30- $\mu$ m mouse striatal and nigral sections as well as human nigral sections as described in our previous publications $(75,76)$. For antigen retrieval, $10 \mathrm{mM}$ citrate buffer at pH 7.4 was used for animal samples, whereas citraconic anhydride was used for human samples. Following antigen retrieval, sections were washed with PBS, blocked with blocking buffer ( $2 \%$
BSA, 0.5\% Triton X-100, and 0.05\% Tween-20), and incubated in primary antibodies overnight at $4^{\circ} \mathrm{C}$. Next, sections were washed with PBS, incubated in secondary antibodies for 1 hour, and stained with Hoechst nuclear dye. Finally, sections were mounted on precoated slides and dried overnight before visualization under a fluorescence microscope. Confocal imaging was performed on these sections at ISU's Microscope Facility using a Leica DMIRE2 confocal microscope with a $\times 63$ oil objective. For $Z$-stacking, each optical section consisted of ten to fifteen $0.5-\mu \mathrm{m}$-thick slices.

For ICC on immortalized MMCs and PMCs, $4 \%$ paraformaldehyde (PFA) was used to fix the cells. Next, fixed cells were washed with PBS, blocked using a blocking buffer, and incubated in primary antibodies following the manufacturer's protocol. Following overnight primary antibody incubation, cells were washed with PBS, incubated in secondary antibodies for 75 minutes, and mounted onto slides using Fluoromount Aqueous Mounting Medium (MilliporeSigma). Samples were visualized using an inverted fluorescence microscope (TE-2000U, Nikon). For DAB staining, we followed previously published protocols from our laboratory $(27,38,77)$. The following primary antibodies were used: IBA1 (Wako, 1:1000) (RRID: AB_2314667), IBA1 (Abcam, 1:500) (RRID: AB_870576), Kv1.3 (Alomone Labs, 1:500) (RRID: AB_2040151), Kv1.3 (MilliporeSigma, 1:500)(RRID: AB_11212692), p-Kv1.3 (MilliporeSigma 1:500) (SAB4504254), p38 (Cell Signaling Technology, 1:500) (RRID: AB_330713), and tyrosine hydroxylase (TH) (MilliporeSigma, 1:1000) (RRID: AB_2201526). Alexa dye-conjugated secondary antibodies were used for both ICC and IHC.

Flow cytometry. MMCs (50,000/well) were plated onto 12-well cell culture plates. Cells were treated with $1 \mu \mathrm{g} / \mathrm{mL}$ LPS or $1 \mu \mathrm{M} \alpha \mathrm{Syn}_{\mathrm{Agg}}$ for 24 hours. Following treatment, cells were double washed with HBSS and blocked with $2 \%$ BSA in HBSS for 30 minutes at $37^{\circ} \mathrm{C}$. After blocking, cells were incubated in the Kv1.3 antibody conjugated to FITC (1:1000, in 1\% BSA in HBSS) (RRID: AB_2040147) for 1 hour at $37^{\circ} \mathrm{C}$. Following incubation, any unbound antibody was double washed with HBSS, and the cells were scraped from the plate and suspended in 500 $\mu \mathrm{L}$ HBSS. A FACSCanto Cell Analyzer (BD Biosciences) was used for flow cytometric analysis at ISU's flow cytometry facility.

Duolink PLA. Duolink PLA was performed according to previously published methods (78). Briefly, 10,000 PMCs/well were plated on coverslips in 96-well cell culture plates. Cells were treated with 1 $\mu \mathrm{M} \alpha \mathrm{Syn}_{\mathrm{Agg}}$ for 24 hours, after which the cells were fixed with $4 \%$ PFA, blocked (2\% BSA, 0.5\% Triton X-100, 0.05\% Tween-20 in PBS), and incubated overnight in primary antibody solutions. Following primary antibody incubation, a Duolink In Situ Red Starter Kit (MilliporeSigma; DUO92101) was used according to the manufacturer's protocol. A mouse Fyn monoclonal antibody (Thermo Fisher Scientific, 1:500) (RRID: AB_1074491) and a rabbit Kv1.3 antibody (Alomone Labs, 1:500) (RRID: AB_2040151) were used as primary antibodies. Slides were imaged using a Leica DMIRE2 confocal microscope with a $\times 63$ oil objective. For $Z$-stacking, each optical section consisted of ten to fifteen 0.5 - $\mu$ m-thick slices.

Immunoprecipitation. Immunoprecipitation was performed following our laboratory's previously published protocol (27). Briefly, $5 \times 10^{6}$ microglial cells were homogenized using NP40 lysis buffer. Lysates were incubated overnight with the Fyn antibody (Thermo Fisher Scientific, RRID: AB_1074491) and the Kv1.3 antibody (Alomone Labs, RRID: AB_2040151) separately. Protein G-sepharose 
beads were used for pulldown. The lysates were incubated with the protein G-sepharose beads and then washed 4 times before loading.

Whole-cell patch clamping. PMCs were plated at 100,000150,000 cells per well in 24-well plates for 8-12 hours before stimulation with either $300 \mathrm{ng} / \mathrm{mL}$ LPS or $1 \mu \mathrm{M} \alpha \mathrm{Syn}_{\mathrm{Agg}}$ in the presence or absence of $10 \mu \mathrm{M}$ saracatinib. After a 40 - to 48 -hour stimulation, cells were detached by trypsinization, washed, attached to polyL-lysine-coated glass coverslips, and then studied within 20-90 minutes after plating in the whole-cell mode of the patch-clamp technique with an EPC-10 HEKA amplifier (HEKA Elektronik). B lymphocytes were thawed, plated, and maintained in a tissue culture incubator for 30-60 minutes before recording. All currents were recorded in normal Ringer's solution containing $160 \mathrm{mM} \mathrm{NaCl}, 4.5$ $\mathrm{mM} \mathrm{KCl}, 2 \mathrm{mM} \mathrm{CaCl}_{2}, 1 \mathrm{mM} \mathrm{MgCl}_{2}$, and $10 \mathrm{mM}$ HEPES (adjusted to pH 7.4 and 290-310 mOsm). Patch pipettes were pulled from sodalime glass (micro-hematocrit tubes, Kimble Chase) to resistances of 2-3 $\mathrm{M} \Omega$ when submerged in the bath solution and filled with a KF-based $\mathrm{Ca}^{2+}$-free internal pipette solution containing $145 \mathrm{mM} \mathrm{KF}$, $2 \mathrm{mM} \mathrm{MgCl}, 10 \mathrm{mM}$ HEPES, and $10 \mathrm{mM}$ EGTA (pH 7.2, 290-310 mOsm). On the basis of our previous publication identifying Kir2.1, $\mathrm{Kv} 1.3$ and $\mathrm{KCa} 3.1$ as the major $\mathrm{K}^{+}$channels expressed in cultured microglia (21), recording conditions were set up to isolate Kv1.3 currents. The use of KF avoids "contaminating" Kv currents by calcium-activated $\mathrm{K}^{+}$currents or chloride currents. Currents were elicited with a "use-dependence protocol," involving a train of ten 200ms voltage steps from -80 to $40 \mathrm{mV}$ applied at a frequency of $1 \mathrm{~Hz}$, which identifies Kv1.3 by its characteristic use dependence (e.g., current amplitude declines rapidly when pulsed faster than the channels can recover from inactivation). In $25 \%$ of the cells, we then further pharmacologically confirmed that the current was predominantly carried by Kv1.3 by testing its sensitivity to PAP-1. Cell capacitance, which directly measures the cell-surface area, and access resistance were continuously monitored during recordings. Kv1.3 current density was calculated as the use-dependent current amplitude at 40 $\mathrm{mV}$ divided by the cell capacitance measured for individual cells.

HPLC. HPLC for neurotransmitters was performed following previously published methods (70). Briefly, the left mouse striatum was weighed and lysed using an extraction solution $(0.1 \mathrm{M}$ perchloric acid containing $0.05 \% \mathrm{Na}_{2}$ EDTA and $0.1 \% \mathrm{Na}_{2} \mathrm{~S}_{2} \mathrm{O}_{5}$ ) and isoproterenol (internal standard). A C-18 reversed-phase column isocratically separated dopamine, 3,4-dihydroxyphenylacetic acid (DOPAC), homovanillic acid (HVA), and norepinephrine (NE) using a Dionex Ultimate 3000 HPLC system (pump ISO-3100SD, Thermo Scientific) attached to an electrochemical detection system consisting of the CoulArray Model 5600A (Thermo Fisher Scientific) coupled with an analytical cell (microdialysis cell 5014B) and a guard cell (model 5020) with the 4-channel potentials set at 350, 0, -150, and $220 \mathrm{mV}$. Chromeleon 7 and ESA CoulArray 3.10 HPLC software were used for data acquisition and analysis.

Multiplex cytokine assay. Multiple cytokine assays were performed according to our previous publication (27). Briefly, $40 \mu \mathrm{L}$ treatment media were incubated overnight with $40 \mu \mathrm{L}$ primary antibodies con- jugated with magnetic beads. Following incubation with primary antibodies, the samples were washed, incubated with biotinylated antibody and biotin/streptavidin, and then assayed on a Bio-Plex plate reader (Bio-Rad). Standards were obtained from PeproTech.

Griess assay. Griess assays were performed as we previously described (27). Briefly, after treatment, $50 \mu \mathrm{L}$ medium was incubated with $50 \mu \mathrm{L}$ Griess reagent for 10 minutes, and a plate reader was used to read the absorbance at $540 \mathrm{~nm}$.

Statistics. GraphPad 5.0 was used for statistical analysis, with $P$ values of 0.05 or less considered statistically significant. One-way ANOVA was used for comparing multiple groups. In most cases, Tukey's post hoc analysis was applied. A 2-tailed Student's $t$ test was used when comparing 2 groups.

Study approval. The laboratory animal facility of ISU is fully accredited by the Association for Assessment and Accreditation of Laboratory Animal Care (AAALAC), and all procedures involving animal handling were approved by the IACUC at ISU. Human brain samples were obtained from the brain repositories of the UCD Alzheimer's Disease Center (P30 AG10129) with IRB approval.

\section{Author contributions}

SS, AGK, and HW conceived the study. SS, DR, VS, HMN, JL, EM, and MG developed methodology. SS, DR, VS, HMN, JL, EM, ML, BNP, NS, SM, MN, MG, AA, PA, NP, VS, MA, AC, DH, LWJ, SR, HW, and AGK performed experiments and analyses. SS, HJ, VA, AGK, and HW wrote the original draft of the manuscript. SS, AGK, HJ, SR, VA, HW, and HMN reviewed and edited the manuscript. AGK and HW acquired funding. AGK, HW, SR, and LWJ provided resources.

\section{Acknowledgments}

This study was supported by NIH grant NS100090. Experimental supplies and salaries were also partially supported by other NIH grants (ES026892 and NS088206). This work was also supported in part by NIH grants P30 AG10129 and 1K08NS099474-01 (to SR) to supply AD and DLB samples. We give special thanks to Douglas T. Golenbock of the University of Massachusetts Medical School for providing the MMC cell line and Debra A. Fadool of Florida State University for providing the Kv.1.3 and mutant plasmids. We also acknowledge the Lloyd Endowed Chair and Eminent Scholar and the Armbrust endowment to AGK. We also thank Gary Zenitsky for assistance in preparing this manuscript.

Address correspondence to: Anumantha Kanthasamy, Department of Biomedical Sciences, Iowa State University, Ames, Iowa 50011, USA. Phone: 515.294.2516; Email: akanthas@iastate.edu

SS's present affiliation is: Department of Pathology, Brigham and Women's Hospital, Harvard Medical School, Boston, Massachusetts, USA.

MA's present affiliation is: Department of Genetics and Bioengineering, Alanya Alaaddin Keykubat University, Antalya, Turkey.
1. Doorn KJ, et al. Emerging roles of microglial activation and non-motor symptoms in Parkinson's disease. Prog Neurobiol. 2012;98(2):222-238.

2. Wolters ECh. Non-motor extranigral signs and symptoms in Parkinson's disease. Parkinsonism Relat Disord. 2009;15 Suppl 3:S6-12.

3. Tansey MG, Goldberg MS. Neuroinflammation in Parkinson's disease: its role in neuronal death and implications for therapeutic intervention. Neurobiol Dis. 2010;37(3):510-518.

4. Block ML, Zecca L, Hong JS. Microglia-mediated neurotoxicity: uncovering the molecular mecha- 
nisms. Nat Rev Neurosci. 2007;8(1):57-69.

5. Glass CK, Saijo K, Winner B, Marchetto MC, Gage FH. Mechanisms underlying inflammation in neurodegeneration. Cell.2010;140(6):918-934.

6. Imamura K, Hishikawa N, Sawada M, Nagatsu T, Yoshida M, Hashizume Y. Distribution of major histocompatibility complex class II-positive microglia and cytokine profile of Parkinson's disease brains. Acta Neuropathol. 2003;106(6):518-526.

7. Kim C, et al. Neuron-released oligomeric $\alpha$-synuclein is an endogenous agonist of TLR 2 for paracrine activation of microglia. Nat Commun. 2013;4:1562

8. Gustot A, Gallea JI, Sarroukh R, Celej MS, Ruysschaert JM, Raussens V. Amyloid fibrils are the molecular trigger of inflammation in Parkinson's disease. Biochem J. 2015;471(3):323-333.

9. Codolo G, et al. Triggering of inflammasome by aggregated $\alpha$-synuclein, an inflammatory response in synucleinopathies. PLOS ONE. 2013;8(1):e55375.

10. Panicker N, et al. Fyn kinase regulates misfolded $\alpha$-synuclein uptake and NLRP3 inflammasome activation in microglia. J Exp Med. 2019;216(6):1411-1430.

11. Daniele SG, Béraud D, Davenport C, Cheng $\mathrm{K}$, Yin H, Maguire-Zeiss KA. Activation of MyD88-dependent TLR1/2 signaling by misfolded $\alpha$-synuclein, a protein linked to neurodegenerative disorders. Sci Signal. 2015;8(376):ra45.

12. Lee EJ, et al. Alpha-synuclein activates microglia by inducing the expressions of matrix metalloproteinases and the subsequent activation of protease-activated receptor-1. J Immunol. 2010;185(1):615-623.

13. Chandy KG, et al. A family of three mouse potassium channel genes with intronless coding regions. Science. 1990;247(4945):973-975.

14. Swanson R, et al. Cloning and expression of cDNA and genomic clones encoding three delayed rectifier potassium channels in rat brain. Neuron. 1990;4(6):929-939.

15. Matteson DR, Deutsch C. K channels in T lymphocytes: a patch clamp study using monoclonal antibody adhesion. Nature. 1984;307(5950):468-471.

16. DeCoursey TE, Chandy KG, Gupta S, Cahalan MD. Voltage-gated $\mathrm{K}^{+}$channels in human $\mathrm{T}$ lymphocytes: a role in mitogenesis? Nature. 1984;307(5950):465-468.

17. Koschak A, Bugianesi RM, Mitterdorfer J, Kaczorowski GJ, Garcia ML, Knaus HG. Subunit composition of brain voltage-gated potassium channels determined by hongotoxin-1, a novel peptide derived from Centruroides limbatus venom. JBiol Chem. 1998;273(5):2639-2644.

18. Xu J, et al. The voltage-gated potassium channel Kv1.3 regulates peripheral insulin sensitivity. Proc Natl Acad Sci USA. 2004;101(9):3112-3117.

19. Upadhyay SK, et al. Selective Kv1.3 channel blocker as therapeutic for obesity and insulin resistance. Proc Natl Acad Sci USA. 2013;110(24):E2239-E2248.

20. Gocke AR, et al. Kv1.3 deletion biases T cells toward an immunoregulatory phenotype and renders mice resistant to autoimmune encephalomyelitis. JImmunol. 2012;188(12):5877-5886.

21. Fadool DA, et al. Kv1.3 channel gene-targeted dele- tion produces "Super-Smeller Mice" with altered glomeruli, interacting scaffolding proteins, and biophysics. Neuron. 2004;41(3):389-404.

22. Fordyce CB, Jagasia R, Zhu X, Schlichter LC. Microglia Kv1.3 channels contribute to their ability to kill neurons. J Neurosci. 2005;25(31):7139-7149.

23. Nguyen HM, et al. Differential Kv1.3, KCa3.1, and Kir2.1 expression in "classically" and "alternatively” activated microglia. Glia. 2017;65(1):106-121.

24. Di Lucente J, Nguyen HM, Wulff H, Jin LW, Maezawa I. The voltage-gated potassium channel $\mathrm{Kv} 1.3$ is required for microglial pro-inflammatory activation in vivo. Glia. 2018;66(9):1881-1895.

25. Rangaraju S, Gearing M, Jin LW, Levey A. Potassium channel Kv1.3 is highly expressed by microglia in human Alzheimer's disease. J Alzheimers Dis. 2015;44(3):797-808.

26. Maezawa I, et al. Kv1.3 inhibition as a potential microglia-targeted therapy for Alzheimer's disease: preclinical proof of concept. Brain. 2018;141(2):596-612.

27. Panicker N, et al. Fyn kinase regulates microglial neuroinflammatory responses in cell culture and animal models of Parkinson's disease. J Neurosci. 2015;35(27):10058-10077.

28. Schmitz A, et al. Design of PAP-1, a selective small molecule Kv1.3 blocker, for the suppression of effector memory T cells in autoimmune diseases. Mol Pharmacol. 2005;68(5):1254-1270.

29. Alvarez-Erviti L, Couch Y, Richardson J, Cooper JM, Wood MJ. Alpha-synuclein release by neurons activates the inflammatory response in a microglial cell line. Neurosci Res. 2011;69(4):337-342.

30. Khodr CE, et al. An $\alpha$-synuclein AAV gene silencing vector ameliorates a behavioral deficit in a rat model of Parkinson's disease, but displays toxicity in dopamine neurons. Brain Res. 2011;1395:94-107.

31. Ekstrand MI, et al. Progressive parkinsonism in mice with respiratory-chain-deficient dopamine neurons. Proc Natl Acad Sci USA. 2007;104(4):1325-1330.

32. Gordon R, et al. Inflammasome inhibition prevents $\alpha$-synuclein pathology and dopaminergic neurodegeneration in mice. Sci Transl Med 2018;10(465):eaah4066.

33. Vila M, Vukosavic S, Jackson-Lewis V, Neystat M, Jakowec M, Przedborski S. Alpha-synuclein up-regulation in substantia nigra dopaminergic neurons following administration of the parkinsonian toxin MPTP. J Neurochem. 2000;74(2):721-729.

34. Kowall NW, Hantraye P, Brouillet E, Beal MF, McKee AC, Ferrante RJ. MPTP induces alpha-synuclein aggregation in the substantia nigra of baboons. Neuroreport. 2000;11(1):211-213.

35. Kang JA, et al. Epigenetic regulation of Kcna3-encoding Kv1.3 potassium channel by cereblon contributes to regulation of $\mathrm{CD} 4^{+} \mathrm{T}$-cell activation. Proc Natl Acad Sci USA. 2016;113(31):8771-8776.

36. D'Addario M, Arora PD, McCulloch CA. Role of p38 in stress activation of Sp1. Gene. 2006;379:51-61.

37. Saha RN, Jana M, Pahan K. MAPK p38 regulates transcriptional activity of NF-kappaB in primary human astrocytes via acetylation of p65. J Immunol. 2007;179(10):7101-7109.

38. Gordon R, et al. Protein kinase C $\delta$ upregulation in microglia drives neuroinflammatory responses and dopaminergic neurodegeneration in experimental models of Parkinson's disease. Neurobiol Dis. 2016;93:96-114.

39. Holmes TC, Fadool DA, Levitan IB. Tyrosine phosphorylation of the Kv1.3 potassium channel. J Neurosci. 1996;16(5):1581-1590.

40. Bowlby MR, Fadool DA, Holmes TC, Levitan IB. Modulation of the Kv1.3 potassium channel by receptor tyrosine kinases. J Gen Physiol. 1997;110(5):601-610.

41. Chung I, Schlichter LC. Regulation of native Kv1.3 channels by cAMP-dependent protein phosphorylation. Am J Physiol. 1997;273(2 Pt 1):C622-C633.

42. Cook KK, Fadool DA. Two adaptor proteins differentially modulate the phosphorylation and biophysics of Kv1.3 ion channel by SRC kinase. J Biol Chem. 2002;277(15):13268-13280.

43. Yamada E, Bastie CC. Disruption of Fyn SH3 domain interaction with a proline-rich motif in liver kinase $\mathrm{B} 1$ results in activation of AMP-activated protein kinase. PLOS ONE. 2014;9(2):e89604.

44. Notredame C, Higgins DG, Heringa J. T-Coffee: a novel method for fast and accurate multiple sequence alignment. J Mol Biol. 2000;302(1):205-217.

45. Sarkar S, et al. Characterization and comparative analysis of a new mouse microglial cell mode for studying neuroinflammatory mechanisms during neurotoxic insults. Neurotoxicology. 2018;67:129-140

46. Kundu-Raychaudhuri S, Chen YJ, Wulff H, Raychaudhuri SP. Kv1.3 in psoriatic disease: PAP-1, a small molecule inhibitor of Kv1.3 is effective in the SCID mouse psoriasis--xenograft model. JAutoimmun. 2014;55:63-72.

47. Beeton C, et al. Kv1.3 channels are a therapeutic target for T cell-mediated autoimmune diseases. Proc Natl Acad Sci USA. 2006;103(46):17414-17419.

48. Chen YJ, Nguyen HM, Maezawa I, Jin LW, Wulff $\mathrm{H}$. Inhibition of the potassium channel Kv1.3 reduces infarction and inflammation in ischemic stroke. Ann Clin Transl Neurol. 2018;5(2):147-161.

49. Pereira LE, Villinger F, Wulff H, Sankaranarayanan A, Raman G, Ansari AA. Pharmacokinetics, toxicity, and functional studies of the selective Kv1.3 channel blocker 5-(4-phenoxybutoxy)psoralen in rhesus macaques. Exp Biol Med (Maywood). 2007;232(10):1338-1354.

50. Morrison HW, Filosa JA. A quantitative spatiotemporal analysis of microglia morphology during ischemic stroke and reperfusion. $\mathrm{J} \mathrm{Neu}$ roinflammation. 2013;10:4.

51. Karuppagounder SS, Brahmachari S, Lee Y, Dawson VL, Dawson TM, Ko HS. The c-Abl inhibitor nilotinib, protects dopaminergic neurons in a preclinical animal model of Parkinson's disease. Sci Rep. 2014;4:4874.

52. Nygaard HB, van Dyck CH, Strittmatter SM. Fyn kinase inhibition as a novel therapy for Alzheimer's disease. Alzheimers Res Ther. 2014;6(1):8.

53. McGeer PL, Itagaki S, Boyes BE, McGeer EG. Reactive microglia are positive for HLA-DR in the substantia nigra of Parkinson's and Alzheimer's disease brains. Neurology. 1988;38(8):1285-1291.

54. Perry VH. Innate inflammation in Parkinson's disease. Cold Spring Harb Perspect Med. 
2012;2(9):a009373.

55 . Mao X, et al. Pathological $\alpha$-synuclein transmission initiated by binding lymphocyte-activation gene 3. Science. 2016;353(6307):aah3374.

56 . Harischandra DS, et al. Manganese promotes the aggregation and prion-like cell-to-cell exosomal transmission of $\alpha$-synuclein. Sci Signal. 2019;12(572):eaau4543.

57. Chimote AA, Kuras Z, Conforti L. Disruption of kv1.3 channel forward vesicular trafficking by hypoxia in human T lymphocytes. J Biol Chem. 2012;287(3):2055-2067.

58. Li H, Lemay S, Aoudjit L, Kawachi H, Takano T. SRC-family kinase Fyn phosphorylates the cytoplasmic domain of nephrin and modulates its interaction with podocin. J Am Soc Nephrol. 2004;15(12):3006-3015.

59. Cahalan MD, Chandy KG. The functional network of ion channels in T lymphocytes. Immunol Rev. 2009;231(1):59-87.

60. Wang T, et al. Activated T-cells inhibit neurogenesis by releasing granzyme B: rescue by Kv1.3 blockers. J Neurosci. 2010;30(14):5020-5027.

61. Liu J, Xu C, Chen L, Xu P, Xiong H. Involvement of Kv1.3 and p38 MAPK signaling in HIV-1 glycoprotein 120-induced microglia neurotoxicity. Cell Death Dis. 2012;3:e254.

62. Rangaraju S, et al. A systems pharmacology-based approach to identify novel Kv1.3 channel-dependent mechanisms in microglial activation. J Neuroinflammation. 2017;14(1):128.

63. Tubert C, et al. Decrease of a current mediated by Kv1.3 channels causes striatal cholinergic interneuron hyperexcitability in experimental Parkinsonism. Cell Rep. 2016;16(10):2749-2762.

64. Branch SY, Chen C, Sharma R, Lechleiter JD, Li S, Beckstead MJ. Dopaminergic neurons exhibit an age-dependent decline in electrophysiological parameters in the MitoPark mouse model of Parkinson's disease. J Neurosci. 2016;36(14):4026-4037.

65 . He T, et al. Epigenetic regulation of voltage-gated potassium ion channel molecule Kv1.3 in mechanisms of colorectal cancer. Discov Med. 2017;23(126):155-162.

66. Brevet M, Haren N, Sevestre H, Merviel P, Ouadid-Ahidouch H. DNA methylation of $\mathrm{K}(\mathrm{v}) 1.3$ potassium channel gene promoter is associated with poorly differentiated breast adenocarcinoma. Cell Physiol Biochem. 2009;24(1-2):25-32.

67. Jiménez-Pérez L, et al. Molecular determinants of Kv1.3 potassium channels-induced proliferation. J Biol Chem. 2016;291(7):3569-3580.

68. Gordon R, Hogan CE, Neal ML, Anantharam V, Kanthasamy AG, Kanthasamy A. A simple magnetic separation method for high-yield isolation of pure primary microglia. J Neurosci Methods. 2011;194(2):287-296.

69. Sarkar S, et al. Rapid and refined CD11b magnetic isolation of primary microglia with enhanced purity and versatility. J Vis Exp. 2017;(122):55364

70. Sarkar S, et al. Manganese exposure induces neuroinflammation by impairing mitochondrial dynamics in astrocytes. Neurotoxicology. 2018;64:204-218.
71. Sarkar S, et al. Manganese activates NLRP3 inflammasome signaling and propagates exosomal release of ASC in microglial cells. Sci Signal. 2019;12(563):eaat9900.

72. Sarkar S, et al. Molecular Signatures of neuroinflammation induced by $\alpha$ Synuclein aggregates in microglial cells. Front Immunol. 2020;11:33.

73. Halle A, et al. The NALP3 inflammasome is involved in the innate immune response to amyloid-beta. Nat Immunol. 2008;9(8):857-865.

74. Seo J, Ottesen EW, Singh RN. Antisense methods to modulate pre-mRNA splicing. Methods $\mathrm{Mol}$ Biol. 2014;1126:271-283.

75. Ghosh A, et al. Mitoapocynin treatment protects against neuroinflammation and dopaminergic neurodegeneration in a preclinical animal model of Parkinson's disease. J Neuroimmune Pharmacol. 2016;11(2):259-278.

76. Ghosh A, et al. Anti-inflammatory and neuroprotective effects of an orally active apocynin derivative in pre-clinical models of Parkinson's disease. J Neuroinflammation. 2012;9:241.

77. Langley M, et al. Mito-Apocynin Prevents Mitochondrial Dysfunction, Microglial Activation, Oxidative Damage, and Progressive Neurodegeneration in MitoPark Transgenic Mice. Antioxid Redox Signal. 2017;27(14):1048-1066.

78. Sarkar S, et al. Mitochondrial impairment in microglia amplifies NLRP3 inflammasome proinflammatory signaling in cell culture and animal models of Parkinson's disease. NPJ Parkinsons Dis. 2017;3:30. 